NASA-CR-205129

\title{
A simulated spectrum of convectively generated gravity waves: Propagation from the tropopause to the mesopause and effects on the middle atmosphere
}

\author{
M. Joan Alexander \\ Department of Atmospheric Sciences, University of Washington, Seattle
}

\begin{abstract}
This work evaluates the interaction of a simulated spectrum of convectively generated gravity waves with realistic middle atmosphere mean winds. The wave spectrum is derived from the nonlinear convection model described by Alexander et al. [1995] that simulated a two-dimensional midlatitude squall line. This spectrum becomes input to a linear ray tracing model for evaluation of wave propagation as a function of height through climatological background wind and buoyancy frequency profiles. The energy defined by the spectrum as a function of wavenumber and frequency is distributed spatially and temporally into wave packets for the purpose of estimating wave amplitudes at the lower boundary of the ray tracing model. A wavelet analysis provides an estimate of these wave packet widths in space and time. Without this redistribution of energies into wave packets the Fourier analysis alone inaccurately assumes the energy is evenly distributed throughout the storm model domain. The growth with height of wave amplitudes is derived from wave action flux conservation coupled to a convective instability saturation condition. Mean flow accelerations and wave energy dissipation profiles are derived from this analysis and compared to parameterized estimates of gravity wave forcing, providing a measure of the importance of the storm source to global gravity wave forcing. The results suggest that a single large convective storm system like the simulated squall line could provide a significant fraction of the zonal mean gravity wave forcing at some levels, particularly in the mesosphere. The vertical distributions of mean flow acceleration and energy dissipation do not much resemble the parameterized profiles in form because of the peculiarities of the spectral properties of the waves from the storm source. The ray tracing model developed herein provides a tool for examining the role of convectively generated waves in middle atmosphere physics.
\end{abstract}

\section{Introduction}

Gravity waves transport energy and momentum from the troposphere to the middle atmosphere where, it is widely recognized, they can have a profound effect on the general circulation patterns, temperature structure, and spatial distributions of mixing ratios of the atmospheric gases. The importance of wave drag and diffusion in the middle atmosphere was clearly demonstrated in zonal mean model studies in the 1980 s [e.g. Holton, 1982, 1983; Dunkerton, 1982; Garcia and Solomon, 1985] that utilized the gravity wave parameterization developed by Lindzen [1981]. Lindzen's parameterization required assumptions about the phase speeds and source distributions of gravity waves which are to date still not well characterized. Holton's [1982] work, as well

Copyright 1996 by the American Geophysical Union.

Paper number 95JD02046.

0148-0227/96/95JD-02406\$05.00 as that of Matsuno [1982], established the importance of high phase speed waves to explain the observed mean zonal wind structure and thereby stressed the importance of wave sources other than flow over topography.

These wave-driven processes are also important in three-dimensional global circulation models where parameterization of gravity wave effects is complicated by the models' sensitivity to the geographical and temporal distributions of wave sources. Planetary scale waves can be resolved explicitly in these models, however the effects of smaller-scale waves (of the order of $100 \mathrm{~km}$ and less) will likely be treated only via parameterization for some time to come. Orographically excited waves have been successfully parameterized in such models and have been shown to affect circulation in the troposphere as well as the middle atmosphere [Palmer et al., 1986; McFarlane, 1987; Bacmeister, 1995]. Other wave sources have been more difficult to characterize. Specifically, waves excited by convective activity are likely very important in the tropics and southern hemisphere where there are few orographic wave sources, and con- 
vection may be a source of the high phase speed waves known to be important in the mesosphere.

Recent modeling efforts reported by Fovell et al. [1992], Holton and Durran [1993], and Alexander et al. [1995] have described properties of vertically propagating waves generated by deep convection in the form of a two-dimensional squall line. In the work of Alexander et al. [1995; hereafter referred to as AHD] the spectral properties of the waves in the stratosphere in their simulation were characterized and compared to observations. They noted a strong response at high frequencies (corresponding to periods of $8 \mathrm{~min}$ to 1 hour) and at long vertical wavelengths $(6-10 \mathrm{~km})$, which show similarity to observations of stratospheric motions above convective sources [Larsen et al., 1982; Sato, 1993], and which may be characteristic of waves associated with deep convection. Additional modeling studies and observations will eventually clarify the generality of these spectral characteristics.

In this work, the interaction of this spectrum of convectively generated waves with realistic middle atmosphere winds is examined via a linear ray tracing technique which uses conservation of wave action flux to predict wave amplitudes as a function of height. Wave interactions with the mean flow are included as saturation effects when amplitudes exceed convective instability limits and via the filtering effects of critical level absorption and wave reflection. This method is chosen as a means of estimating the importance of the contribution of waves from such a convective source to the estimated global gravity wave forcing. It is unrealistic to extend the domain of the nonlinear simulation of $A H D$ to the altitudes and horizontal distances required to make this estimate. Durran [1995] also highlights the difficulty in using traditional diagnostic methods for evaluating gravity wave dissipation effects in a domain of limited size. The approach here is to take the twodimensional power spectrum derived from the nonlinear model (AHD) as input to a linear wave propagation calculation. The power in this spectrum is distributed into packets of finite width in horizontal distance, $x$, and time, $t$, and the packet widths are estimated from a wavelet analysis of the nonlinear model results. The linear and nonlinear results are tested for consistency below $32-\mathrm{km}$ altitude where the models overlap. The ray tracing model is in many ways simpler than the threedimensional models described by Eckermann [1992] and Marks and Eckermann [1995] that were designed to study global propagation characteristics over the full range of possible gravity wave frequencies. For the spectrum of convectively generated waves considered here, a number of simplifying assumptions are possible for the purpose of estimating the mean flow forcing.

The results of the linear wave propagation include estimates of the mean flow acceleration and energy dissipation rates associated with the input wave spectrum and specified climatological mean wind profiles. Comparison to the spectral gravity wave parameterization of Fritts and $L u$ [1993] for the same mean wind profiles provides an estimate of the importance of the single storm source to global gravity wave forcing and also gives insight into how the peculiar spectral characteristics associated with the convectively generated waves affect the profile of wave drag and dissipation. The method developed here can be used to compare future simulations to the midlatitude case of AHD and also provides an avenue for testing simpler parameterizations of convectively generated waves against this more complete spectral description.

The following section briefly reviews the convection simulation and determination of the two-dimensional power spectrum described by AHD. The method of converting power spectral density to wave amplitude is also derived, including a wavelet analysis of the nonlinear model results to estimate wave energy packet dimensions in space and time. This then establishes lower boundary conditions for the linear ray tracing analysis described in section 3 , which includes determinations of wave amplitudes as a function of height as well as effects on the mean state. Expressions for mean flow acceleration and rate of wave energy dissipation are derived. Section 4 is devoted to checking the linear propagation model against the full nonlinear model results between 13 and $32 \mathrm{~km}$ where the two models overlap, testing many of the simplifying assumptions in the linear model. In section 5 the wave spectrum interaction with realistic mean wind and buoyancy frequency profiles is examined and compared to the Fritts and $L u$ [1993] parameterized results for the same background state. A concluding summary follows in section 6 .

\section{Determination of Gravity Wave Amplitudes and Propagation Characteristics}

\subsection{The Convection Simulation}

The convectively generated gravity wave spectrum in this analysis is derived from the results of a numerical midlatitude squall line simulation previously described by AHD [Alexander et al., 1995]. Some of the features of the model will be briefly described here. For more details the reader is also referred to previous applications of the squall line model described by Fovell et al. [1992] and Holton and Durran [1993].

The convection simulation from which the gravity wave spectrum is derived is a two-dimensional, nonlinear, compressible, nonhydrostatic squall line model. It resolves a deep stratosphere layer, from the tropopause at $\sim 12 \mathrm{~km}$ up to $32 \mathrm{~km}$ altitude. The full model domain is $840 \mathrm{~km}$ in the horizontal and $32 \mathrm{~km}$ in the vertical and includes wave permeable boundaries at the sides and top. The model reference frame translates eastward at $16 \mathrm{~m} \mathrm{~s}^{-1}$ to track the motion of the storm, keeping it in the center of the domain. Winds in the stratosphere in this model are constant in height at $16 \mathrm{~m} \mathrm{~s}^{-1}$, the same as the reference frame translation speed, and so the stratospheric waves produced by the storm are viewed in their "intrinsic" frame of reference (i.e., the observed wave frequencies are the intrinsic frequencies). A rich 

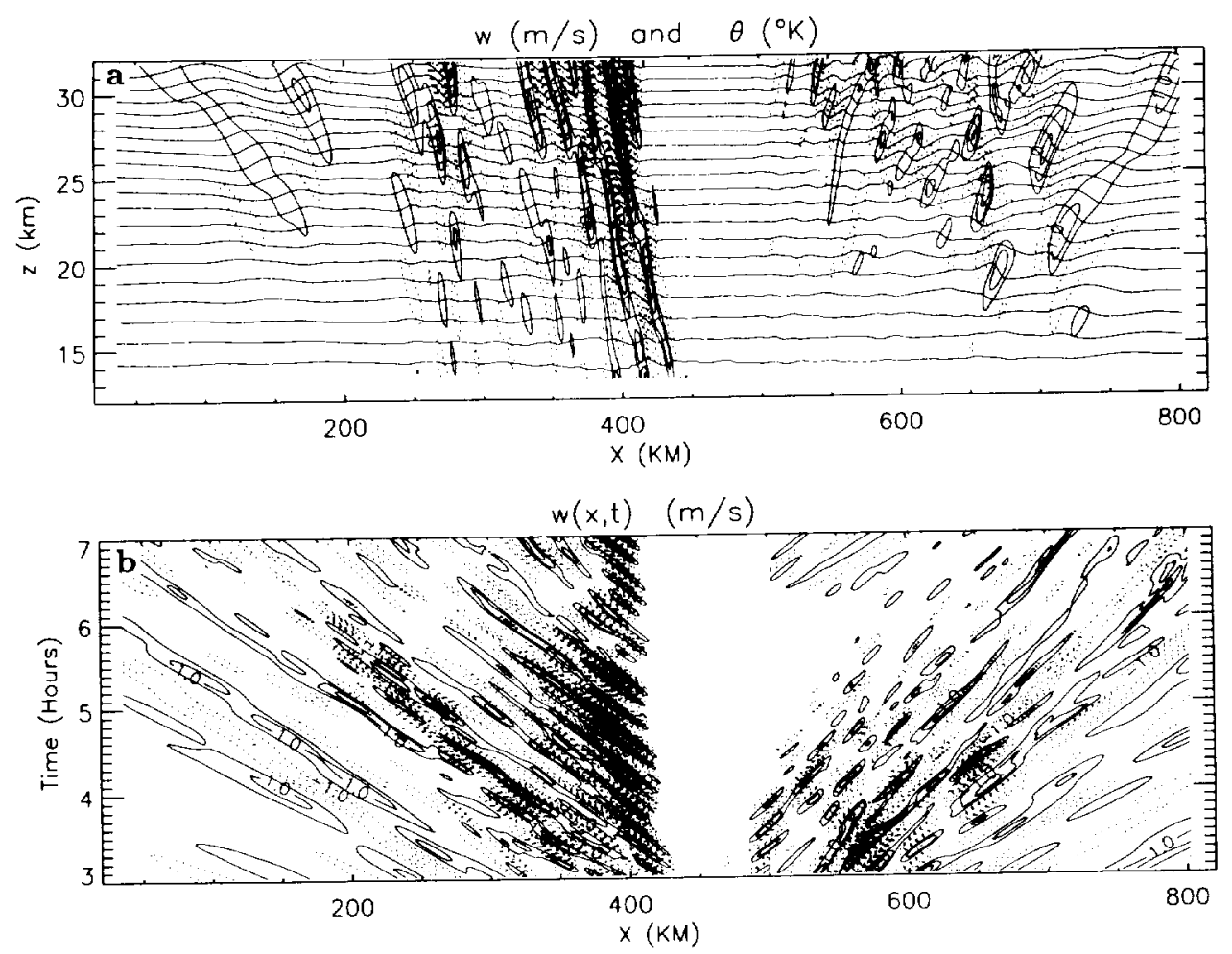

Figure 1. Contours of vertical velocity in the stratospheric portion of the nonlinear convection model. Contours are plotted at $0.5 \mathrm{~m} \mathrm{~s}^{-1}$ intervals. Dotted contours represent negative values. (a) $w(x, z)$ at $t=5$ hours. Horizontal lines represent surfaces of constant potential temperature at $25 \mathrm{~K}$ intervals. The storm center, which is the main source region for these waves, lies in the troposphere below the figure and is located at $x \simeq 420 \mathrm{~km}$. (b) $w(x, t)$ at $z=30 \mathrm{~km}$. The slopes of surfaces of constant phase indicate the intrinsic phase speeds of the wave motions.

spectrum of gravity waves is generated in the stratosphere, as can be seen in Figure 1. Figure 1a shows a single time frame of vertical velocity contours and potential temperature surfaces above $13 \mathrm{~km}$. In Figure $1 \mathrm{~b}$, contours of $w(x, t)$ at $z=30 \mathrm{~km}$ are shown. The slope of the phase surfaces in Figure $1 \mathrm{~b}$ is an indication of the separation east and west of storm center (at $x \sim$ $420 \mathrm{~km}$ ) of eastward and westward propagating waves, respectively, and points to the storm center region as the location of the primary source of the wave energy. A strong preference for forcing of westward propagating waves is observed in this and other similar simulations due to a westward tilt with height of the main tropospheric updraft and the westward propagation of convection cells in the troposphere. These features have been described in earlier work with this model [Fovell et al., 1992; AHD], and have been observed in squall lines in nature [e.g. Houze, 1993]. A spectral analysis of the stratospheric waves was described by AHD and revealed some distinctive spectral signatures which may be characteristic of waves generated by deep convection. AHD describe wave forcing mechanisms and their signatures in the spectral response and also review similarities to observations of wave motions above convective sources.

\subsection{Fourier Spectral Analysis}

The two-dimensional power spectrum in horizontal wavenumber and intrinsic frequency $P(k, \omega)$ computed from the simulation results of AHD will be used as lower boundary input to the linear propagation analysis to follow. The spectrum shown in Figure 2 separates power into eastward and westward phase with positive and negative frequencies, respectively. The power in this spectrum defines wave energies at an altitude of $13 \mathrm{~km}$. The superimposed white contour surrounds regions of the spectrum with power greater than or equal to $5 \times 10^{4}(\mathrm{~m} / \mathrm{s})^{2}(\text { cycle } / \mathrm{m})^{-1}(\text { cycle } / \mathrm{s})^{-1}$. Regions outside this contour with power less than this threshold will be excluded from the following analysis. The contour includes over $93 \%$ of the total energy in the spectrum, and spectral points outside this region are very likely heavily contaminated by spectral bias from regions of high power. Each pixel inside the white contour will be treated as a monchomatic wave packet. The wave amplitude can be derived from (1) the power spectral density at that point, together with (2) the definition of the wave packet width in time and space, and (3) an estimate of power aliasing to other regions of the spec- 


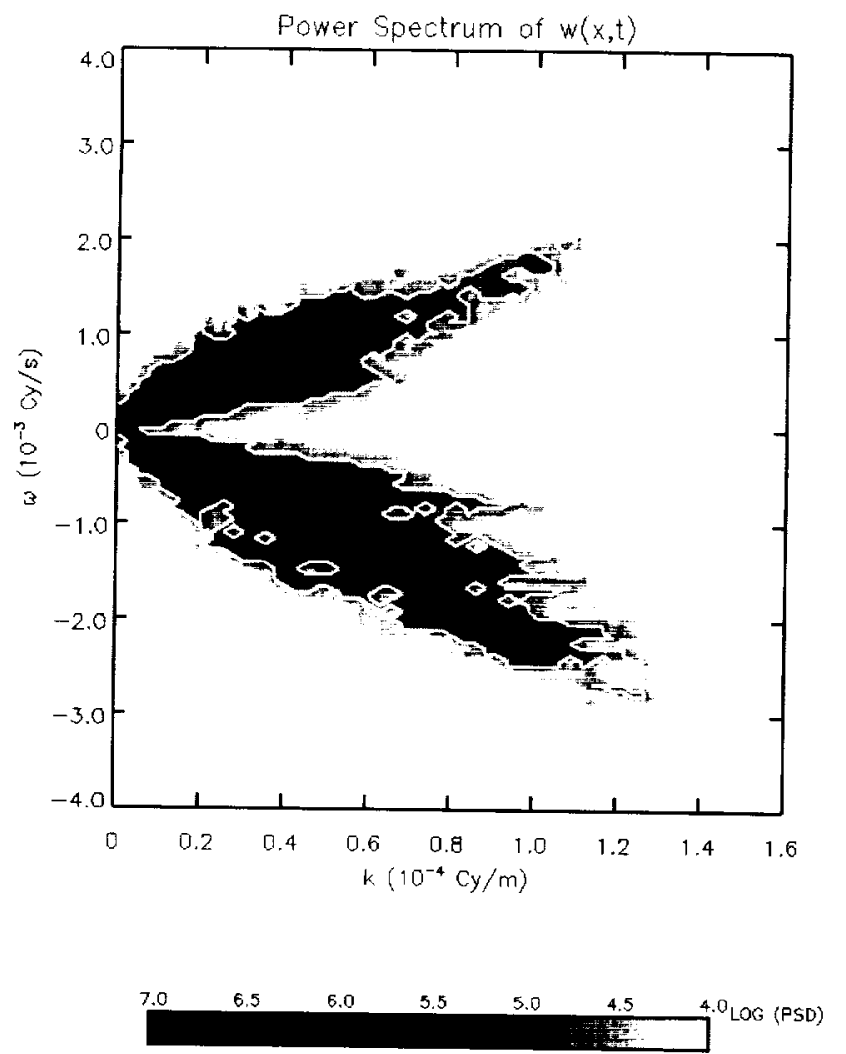

Figure 2. Two-dimensional power spectrum of the vertical velocity field as a function of horizontal wavenumber and intrinsic frequency. The spectrum is derived from the two-dimensional Fourier transform of the field $w(x, t)$ like that shown in Figure 1b. Spectra at each altitude in the model have been averaged by using an $\exp [-(z-13 \mathrm{~km}) / H]$ weighting factor such that the spectral power represents amplitudes at $13 \mathrm{~km}$ near the tropopause.

trum. Parameter (2) will be estimated from a wavelet analysis of the vertical velocity field described in the next section.

The power spectrum in Figure 2 resolves wavenumbers and frequencies,

$$
\begin{array}{cc}
k=\frac{n}{\Delta x N_{x}} & n=0,1, \ldots, \frac{N_{x}}{2} \\
\omega=\frac{ \pm n}{\Delta t N_{t}} & n=0,1, \ldots, \frac{N_{t}}{2}
\end{array}
$$

with $\Delta x=1.5 \mathrm{~km}, N_{x}=256, \Delta t=2 \min$ and $N_{t}=$ 128. So pixel dimensions are $\Delta k=2.6 \times 10^{-6}$ cycle $\mathrm{m}^{-1}$ and $\Delta \omega=6.5 \times 10^{-5}$ cycle $\mathrm{s}^{-1}$. Wave energy with wavenumbers and frequencies smaller than the minimum nonzero values $(k<\Delta k$ and $\omega<\Delta \omega)$ is also omitted from the linear propagation analysis but represents less than $0.2 \%$ of the total energy in the spectrum.

The power spectral density in Figure 2 can be related to the vertical velocity amplitude (in $\mathrm{m} \mathrm{s}^{-1}$ ) if it is assumed that a given pixel represents a wave mode with the frequency and wavenumber associated with that pixel. The two-dimensional power spectral density $P_{k \omega}$ is given by

$$
P_{k \omega}=\frac{2 \Delta x \Delta t}{N_{x} N_{t}}\left|W_{k \omega}\right|^{2},
$$

where $W_{k w}$ is the discrete Fourier transform of $w(x, t)$ at wavenumber $k$ and frequency $\omega$. The power at $(k, \omega)$ describes the mean square amplitude of the wave with those characteristics averaged over the $(x, t)$ domain,

$$
P_{k \omega}=\frac{1}{2}\left|A_{k \omega}\right|^{2} \Delta x N_{x} \Delta t N_{t},
$$

or

$$
A_{k \omega}=\left[2 P_{k \omega} \Delta k \Delta \omega\right]^{1 / 2}
$$

This amplitude would be exactly the wave amplitude if the assumptions (inherent in Fourier analysis) of stationarity throughout the $(x, t)$ domain and periodicity on the $x$ and $t$ intervals were both satisfied. On the contrary, both of these assumptions are violated, as can be seen in Figure 1. There is significant spatial and temporal variation in the spectral properties. The waves can be thought of as concentrated in wave packets in both space and time; i.e., the areal extent of a given mode over the $(x, t)$ domain is limited in both dimensions. These effects make the amplitude predicted from (2) smaller than the true amplitude at any point in $(x, t)$. The power $P_{k w}$ in equation (2) must be adjusted by two multiplicative factors:

1. The first factor is equal to the areal extent of the whole domain divided by the energy-weighted areal extent of the wave packet. This factor arises because the spectrum gives a measure of the mean square amplitude in the domain, not the amplitude in the wave packet.

2. The wave packet envelope can be thought of as a taper function which creates bias in the spectrum, reducing the value of the power at the peaks in the spectrum and spreading that power over a broad range of wavelengths. For a rectangular wave packet the bias would be described by the Fejer kernel [Percival and Walden, 1993, Section 6.4]. The power at the central peak of the kernel is proportional to the length of the window, so this second factor is also proportional to the ratio of the domain area to the envelope area, just as in factor 1. The proportionality constant will vary between 1 and 2 depending on the shape of the wave packet envelope. A value of 1 corresponding to a rectangular shape is assumed for simplicity.

Thus if the wave packets were rectangular in shape, and covered an area $n_{x} n_{t}$ within the entire domain (area $N_{x} N_{t}$ ), then the wave amplitude within that packet becomes

$$
A_{k \omega}=\left[2 P_{k \omega}\left(\frac{N_{x} N_{t}}{n_{x} n_{t}}\right)^{2} \Delta k \Delta \omega\right]^{1 / 2},
$$

with one factor of $\left(N_{x} N_{t} / n_{x} n_{t}\right)$ from each of factors 1 and 2. This equation will be used to estimate amplitudes at the lower boundary for the purpose of predicting breaking levels for each mode in the ray tracing analysis. Parseval's theorem, however, demands that the total power in the spectrum equal the power in the original signal integrated over the $(x, t)$ domain, 


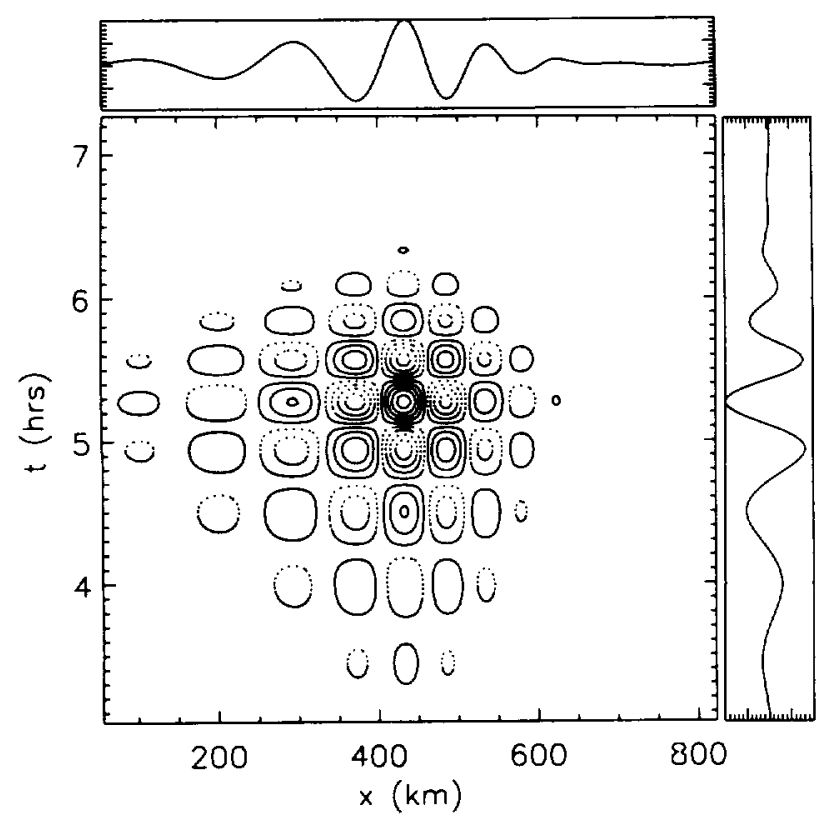

Figure 3. An example of the wavelet basis functions centered in the analysis domain $(x, t)$. Peaks and valleys are plotted with solid and dotted contours, respectively. This is the $96-\mathrm{km}$ wavelength and $32-\mathrm{min}$ period case. The panels along the top and side show cross sections in $x$ and $t$ through the center.

$$
\sum_{k, \omega} P(k, \omega)=\Delta x \Delta t \sum_{x, t}|w(x, t)|^{2} .
$$

Therefore the wave packet amplitude adjustments in (3) must be removed prior to evaluating any net effects of the waves on the mean flow to conserve energy. Wave packet dimensions will be estimated via the wavelet analysis described below.

\subsection{Wavelet Analysis}

Wavelet analysis is a spectral analysis technique that retains information about the spatial/temporal location of variations in the spectral power. Without this information the amplitudes derived solely from the Fourier analysis cannot be used to predict realistic breaking levels. For an energy-conserving orthogonal wavelet set, the spatial/temporal information is gained at the expense of spectral resolution: Given a 128-point time array, the number of resolved frequencies in the wavelet spectrum is only $\log _{2}(128)=7$, compared to the 64 nonzero frequencies obtained from a Fourier analysis. Therefore wavelet analysis cannot replace the Fourier analysis for our purposes but can provide a rough estimate of the spatial/temporal extent of a given wavenumber/frequency signal and thus an estimate of an effective wave packet width.

The $512 \times 128$ array of $w(x, t)$ with $1.5-\mathrm{km}$ and 2min resolution, as shown in Figure $1 \mathrm{~b}$, is examined via wavelet analysis. A two-dimensional, orthogonal wavelet transform is applied and an energy spectrum computed as the square of the resulting wavelet coefficients. Figure 3 shows an example of the wavelet basis functions employed here. The wavelet transform utilizes the set of Daubechies wavelet filters with 20 coefficients summarized in the Numerical Recipes subroutines "pwtset" and "pwt" [Press et al., 1993, sec. 13.10]. The set of basis functions employed in the analysis consists of translations and dilations/contractions of the function shown in Figure 3. The wavelengths and periods resolved in the analysis are summarized in Table 1 , as well as the fractional energy contained in each of these modes. For each point in Table 1, a corresponding array of energy as a function of $(x, t)$ can be produced. The resolutions in $x$ and $t$ of each energy array are equal to the wavelength and period of the mode, respectively. Four examples are shown in Figure 4 for the four modes containing the largest fractional energy. These modes are also highlighted in boldface in Table 1. Together these four modes comprise $64 \%$ of the total energy in the spectrum. Note that the left and right halves of the figure display properties of the westward and eastward propagating waves, respectively. The contours describe how energy for the resolved mode with wavelength and period $\left(\lambda_{x}, T\right)$ is distributed in $(x, t)$. When these figures are compared to Figure $1 \mathrm{~b}$, it can be seen that the wavelet analysis describes how energy in a given spectral mode is localized in $(x, t)$.

Table 1. Percent Energy in the Wavelet Spectrum as a Function of Horizontal Wavelength and Period

\begin{tabular}{|c|c|c|c|c|c|c|c|c|c|}
\hline \multirow[b]{2}{*}{$\mathrm{T}, \mathrm{min}$} & \multicolumn{9}{|c|}{$\lambda_{z}, \mathrm{~km}$} \\
\hline & 768 & 384 & 192 & 96 & 48 & 24 & 12 & 6 & 3 \\
\hline 256 & 0.11 & $<0.01$ & 0.01 & 0.03 & 0.04 & 0.06 & 0.02 & $<0.01$ & $<0.01$ \\
\hline 128 & 0.04 & $<0.01$ & 0.02 & 0.05 & 0.06 & 0.08 & 0.03 & $<0.01$ & $<0.01$ \\
\hline 64 & 0.04 & 0.05 & 0.20 & 1.50 & 1.73 & 0.44 & 0.07 & 0.01 & $<0.01$ \\
\hline 32 & 0.02 & 0.04 & 0.20 & 1.46 & 9.46 & 6.01 & 1.33 & 0.08 & 0.04 \\
\hline 16 & $<0.01$ & $<0.01$ & 0.02 & 0.17 & 4.41 & 15.83 & 10.98 & 0.33 & $<0.01$ \\
\hline 8 & $<0.01$ & $<0.01$ & $<0.01$ & 0.02 & 0.24 & 5.52 & 29.16 & 5.47 & 0.05 \\
\hline 4 & $<0.01$ & $<0.01$ & $<0.01$ & 0.02 & 0.07 & 0.20 & 1.25 & 2.77 & 0.12 \\
\hline
\end{tabular}



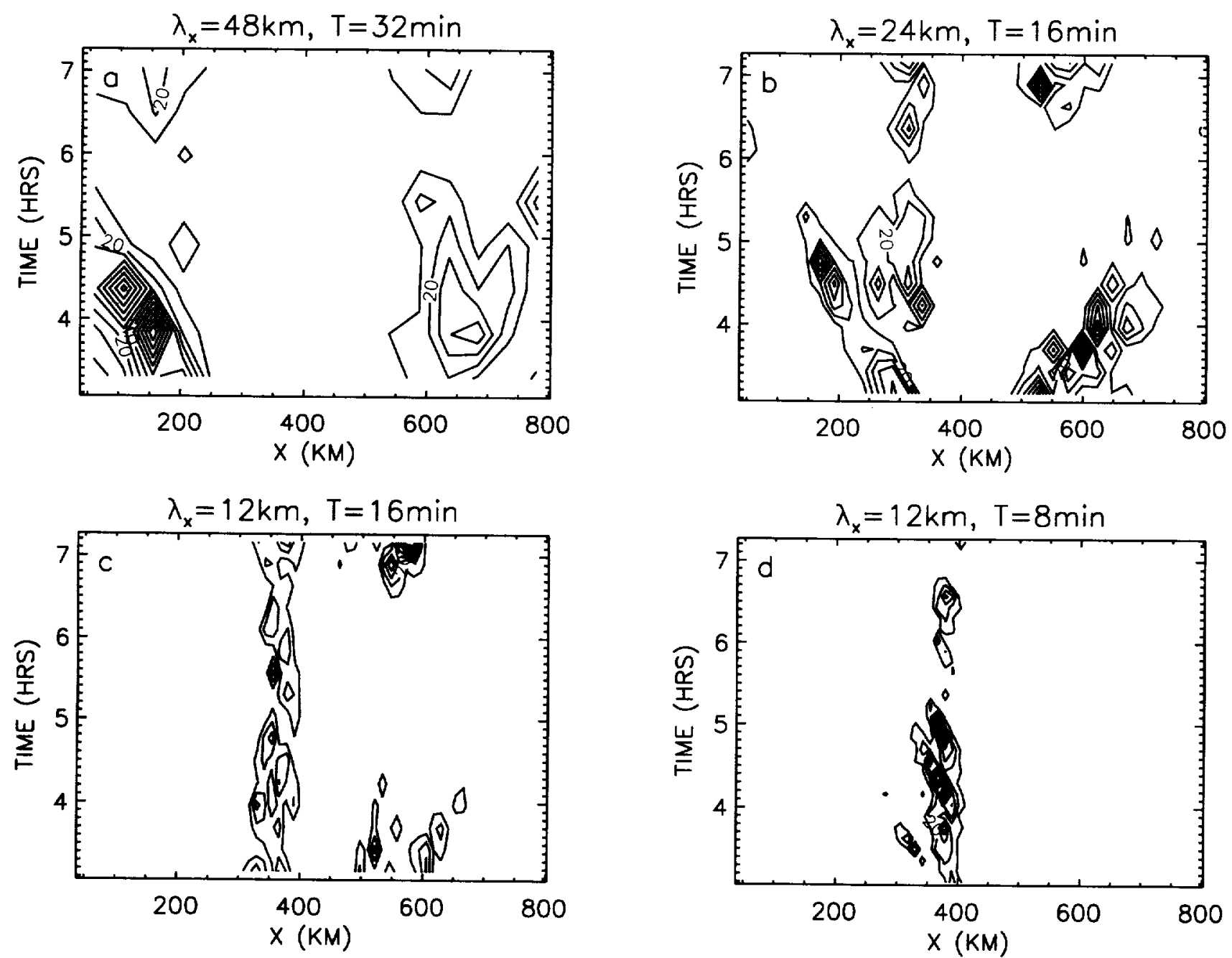

Figure 4. Energy distributions in $(x, t)$ from the wavelet analysis for the four modes in boldface in Table 1. These four modes contain the largest fractional energies in the wavelet spectrum (Table 1). Maps like these show how spectral energy at a given $(k, \omega)$ is distributed in $(x, t)$. Comparison to Figure $1 \mathrm{~b}$ shows how the wavelet analysis captures the spatial/temporal variability in the spectral modes. The contours label energy distribution as percent of maximum at $10 \%$ intervals.

Spatially, Figure 4 shows that energy tends to be tightly constrained within narrow regions of $x$. The span of the distribution in $x$ can also be seen to roughly vary with the wavelength of the mode. The relationship between the energy distribution in time and the wave period is not so obvious. To develop an objective estimate of wave packet widths in space and time, the following method was applied to each energy distribution in Table 1 (like those shown in Figure 4). (Note, however, that the energy arrays representing the longest resolved period and wavelength (top row and first column of Table 1) are omitted because these arrays only resolve a single point in $t$ and $x$, respectively, and contain very little energy anyway.) East and west halves of the domain are analyzed separately to separate eastward and westward propagating modes.

To estimate equivalent packet width in $x$, the energy density for each mode $E_{k \omega}(x, t)$ is summed over time:

$$
E_{k \omega}^{\prime}\left(x_{i}\right)=\sum_{j} E_{k \omega}\left(x_{i}, t_{j}\right)
$$

where $i$ and $j$ are dummy indices representing the discrete grid points in the wavelet energy arrays. The wave packet width $x_{W P}$ is then estimated as the width of a box whose length is equal to the maximum energy in $E_{k w}^{\prime}\left(x_{i}\right)$ and whose area is the total energy in the mode, or

$$
x_{W P}=\frac{\sum_{j} \sum_{i} E_{k \omega}\left(x_{i}, t_{j}\right)}{\max \left[E_{k \omega}^{\prime}\left(x_{i}\right)\right]}
$$

Figure 5 illustrates this definition of wave packet width. The equivalent width $x_{W P}$ is plotted versus wavelength in Figure 6a. An energy weighted least squares straight line fit is computed and overplotted as the solid line in Figure 6a. The correlation coefficient is 0.91 , with slope $=1.24 \pm 0.06$ and intercept $=27 \pm 2 \mathrm{~km}$. Fits to eastward and westward propagating modes were not significantly different, so both are included in Figure 6. The dashed lines show the $95 \%$ confidence limits to the weighted linear fit. The dotted line represents the resolution of the wavelet analysis. These packet widths 


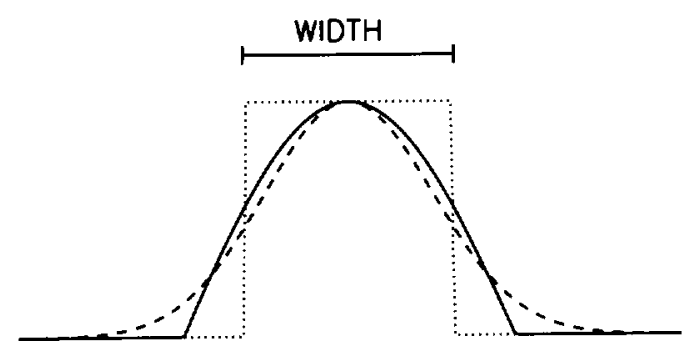

Figure 5. Illustration of the definition of wave packet width. The vertical axis is energy per unit distance, and the horizontal axis is distance. Packet widths $x_{W P}$ are determined as the width of a box (dotted line) whose height is the maximum of the energy distribution and whose area is the total energy in the mode $(k, \omega)$. This width is approximately equal to the full width at half maximum of a Gaussian shaped packet with the same amplitude (dashed line). The cosine envelope with wavelength equal to $\pi x_{W P}$ (solid line) also encloses the same area and is used to reconstruct the wind and temperature fields at a given height as in Figures 7 and 17 .

are fairly narrow compared to the wavelength of the wave they enclose, casting some doubt on the use of the simple oscillatory form assumed in $\boldsymbol{x}$ to compute derivatives in the linear equations, i.e., $\partial / \partial x=i k$. In section 4 the linear analysis will be tested against the full nonlinear model below $32 \mathrm{~km}$ where they overlap, and the linear treatment in fact produces quite realistic results.

An exactly analogous procedure was performed to determine wave packet widths in time, $t_{W P}$, that are plotted in Figure $6 \mathrm{~b}$ along with the weighted least squares straight line fit and $95 \%$ confidence limits. The correlation coefficient is again 0.91 with slope equal to $1.37 \pm 0.06$ and intercept equal to $0.50 \pm 0.02$ hours.

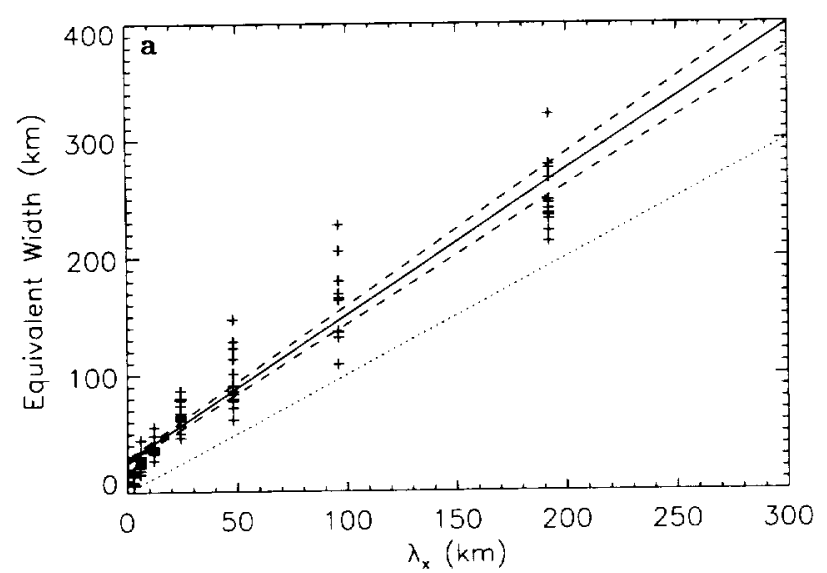

Modes with periods beyond 2 hours are weighted quite low and do not affect the fit, but there is indication of an upper bound to the wave packet width of $\sim 2.5-3$ hours for periods longer than an hour. An upper limit of 3 hours for the wave packet width in time is then chosen as a modification to the straight line fit in Figure 6b.

These results are used to define the amplitude correction factor in (3):

$$
\begin{aligned}
& \frac{n_{x}}{N_{x}}=\frac{x_{W P}}{N_{x} \Delta x} \quad x_{W P}=1.24 \lambda_{x}+27 \mathrm{~km} \\
& \frac{n_{t}}{N_{t}}=\frac{t_{W P}}{N_{t} \Delta t} \quad t_{W P}=1.37 T+0.50 \text { hours }
\end{aligned}
$$

where $\lambda_{x}$ is wavelength in kilometers, and $T$ is wave period in hours. (Note also that these correction factors cannot exceed unity, a value which would indicate a wave packet width equal to the size of the domain.) Table 2 lists values of the correction factor $N_{x} N_{t} / n_{x} n_{t}$ as a function of wavelength and period. Modes containing less than $.01 \%$ of the spectral energy have been omitted. With these correction factors and equation (3) the maximum vertical velocity amplitude at $z=13 \mathrm{~km}$ for any single mode predicted from the power spectrum (Figure 2) is $1.8 \mathrm{~m} \mathrm{~s}^{-1}$. These amplitude corrections are applied for the purpose of determining breaking levels and wave saturation.

\section{Linear Wave Propagation With Saturation}

The following analysis is designed to study the vertical propagation of waves produced in the nonlinear squall line model to altitudes much greater than the current top at $32 \mathrm{~km}$, into the upper stratosphere, mesosphere, and lower thermosphere. The difficulties in ex-

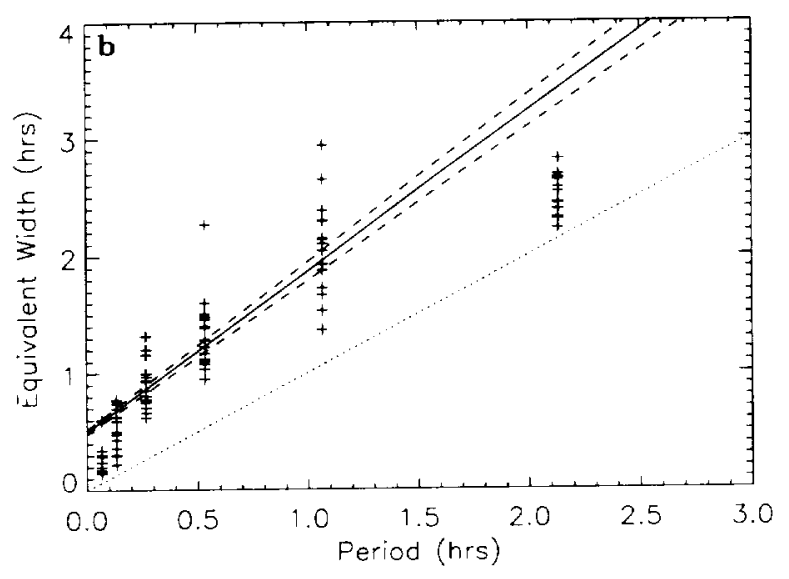

Figure 6. Equivalent wave packet widths in (a) $x$ and (b) $t$, derived from the wavelet analysis plotted as a function of wavelength and period respectively. The result for each mode in Table 1 is represented by a plus symbol. Solid lines show the energy-weighted least squares straight line fits to the wave packet results, which have high correlation coefficients equal to 0.91 . (Note from Table 1 that many of the modes contain very little energy.) Dashed lines show the $95 \%$ confidence limits on these fits, and the dotted line in each panel indicates the resolution limit of the wavelet analysis. The fits define simple relationships between wave packet widths and frequency and wavenumber of the wave which will be used in subsequent analysis. 
Table 2. Wave Packet Correction Factor $\left(N_{a} N_{t}\right) /\left(n_{m} n_{t}\right)$ as a Function of Horizontal Wavelength and Period

\begin{tabular}{rrrrrrrrrrr}
\hline & \multicolumn{10}{c}{$\lambda_{\mathbf{8}}, \mathrm{km}$} \\
\cline { 2 - 10 } $\mathrm{T}, \min$ & 768 & 384 & 192 & 96 & 48 & 24 & 12 & 6 & 3 \\
\hline 256 & 1.0 & & 1.9 & 3.5 & 6.0 & 9.1 & 12.3 & & \\
128 & 1.0 & & 3.6 & 6.6 & 11.1 & 16.9 & 22.9 & & \\
64 & 1.7 & 3.3 & 6.3 & 11.4 & 19.3 & 29.4 & 39.9 & 48.5 & \\
32 & 2.7 & 5.3 & 10.0 & 18.2 & 30.8 & 46.9 & 63.6 & 77.3 & 86.7 \\
16 & & & 14.3 & 25.9 & 43.8 & 66.7 & 90.4 & 110.0 & \\
8 & & & & 32.9 & 55.5 & 84.6 & 114.6 & 139.4 & 156.2 \\
4 & & & & 37.9 & 64.0 & 97.6 & 132.3 & 160.9 & 180.4 \\
\hline
\end{tabular}

tending the mesoscale simulation to study wave-mean flow interactions at these altitudes were described in the introduction. The linear propagation analysis derived here can be used to estimate the effects of the convectively generated waves on the mean flow for realistic middle atmosphere winds, and it also provides a means of comparing the nonlinear simulation results to recent observations at altitudes near the mesopause [Swenson and Mende, 1994; Taylor et al., 1995].

Each point in the power spectrum, $P(k, \omega)$, in Figure 2 is assumed to describe the amplitude of a monochromatic wave $A_{w}(k, \omega)$, at $13 \mathrm{~km}$ altitude, and centered at $x=420 \mathrm{~km}$ at the center of the storm. Note that the frequencies in Figure 2 are intrinsic frequencies at the lower boundary of the linear analysis $(z=13 \mathrm{~km})$, where the mean wind speed is set to $16 \mathrm{~m} \mathrm{~s}^{-1}$ to match conditions in the nonlinear model from which Figure 2 was derived. The power to amplitude conversion is described by equations (3), (4), (5) and (6). Linear gravity wave theory and the polarization relations [Gossard and Hooke, 1975, pp. 97-100] relate this vertical velocity amplitude to the total wave energy per unit density $E$ [Fritts and VanZandt, 1993],

$$
E=\frac{N^{2}}{\omega^{2}} A_{w}^{2}
$$

where $N$ is the buoyancy frequency and $\omega$ is the intrinsic frequency. Similarly, the square of the horizontal velocity amplitude $A_{u}^{2}$ can also be related to $E$ and $A_{w}^{2}$,

$$
A_{u}^{2}=\left(1-\frac{\omega^{2}}{N^{2}}\right) E=\left(\frac{N^{2}}{\omega^{2}}-1\right) A_{w}^{2}
$$

Each wave will follow a ray path, $(X(z))$, described by Lighthill $[1978$, sec. 4.6] as

$$
X(z)=\int_{0}^{z}\left(\frac{d x}{d z^{\prime}}\right)_{r a y} d z^{\prime}
$$

the slope along the ray is defined by the direction of energy propagation relative to a stationary reference frame,

$$
\left(\frac{d x}{d z}\right)_{r a y}=\frac{U(z)+\partial \omega / \partial k}{\partial \omega / \partial m},
$$

where $U(z)$ is the mean wind and $(k, m)$ is the wavenumber vector. The linear nonhydrostatic dispersion relation can be written as

$$
\omega=\frac{ \pm N k}{\left(k^{2}+m^{2}\right)^{1 / 2}}= \pm N \cos \theta
$$

where $\theta$ is the angle at which lines of constant phase lie from the vertical. Equation (11) assumes that $\omega^{2}>>$ $f^{2}$ and $m^{2}>(2 H)^{-2}(f$ is the Coriolis parameter; $H$ is density scale height). The effects of rotation could ecome important when the waves are very near their critical levels. Marks and Eckermann [1995] point out the importance of the density scale height term on the prediction of turning point altitudes; however, for the spectrum considered in this work, neither of these effects is important in estimating the mean flow forcing (see section 5). Differentiation of (11) with respect to $k$ and $m$, and substitution into (10), yields

$$
\left(\frac{d x}{d z}\right)_{r a y}=\mp \frac{k U}{N} \sec ^{2} \theta \csc \theta-\tan \theta .
$$

In general, $U, N$, and $\theta$ all vary with height. This expression describes the slope of the ray path as a function of height and with a simple numerical integration using the trapezoidal rule will define the ray path of the wave. Note that the sign convention used here is $\omega>0(\theta<0)$ for eastward intrinsic phase speeds, $\omega<0(\theta>0)$ for waves with westward intrinsic phase speeds, and $k$ positive definite, which is different from that of Lighthill and accounts for the sign differences in this derivation. The upper sign, then, in these equations refers to eastward and the lower to westward propagating waves in the frame of the storm.

Two conditions on the intrinsic frequency define the maximum height to which waves can propagate:

1. Where the wave phase speed equals the mean wind speed the intrinsic frequency $\omega \rightarrow 0, \theta \rightarrow \mp 90^{\circ}$, and the wave will be absorbed there. This level is traditionally referred to as the critical level. 
2. Where the intrinsic frequency, $\omega \rightarrow N$, and $\cos (\theta) \rightarrow 1$, the vertical wavenumber, $m \rightarrow 0$. Solutions above this level have $m$ imaginary, and so the wave undergoes total internal reflection. In the case of nonzero curvature in the background wind $U(z)$ the solutions can theoretically be modified to permit propagation through this level if the condition $\omega=N$ occurs very near an inflection point in the mean wind profile. This situation is rare enough to neglect, so it will be treated as a simple reflection and such levels will be referred to as turning points.

The ray path integration for each wave is taken only up to the level just below the first critical level or turning point. At and above that height the wave energy is set to zero. Below the critical level or turning point, and in the absence of dissipation, the wave amplitude will change with height according to conservation of wave action flux along a ray [Lighthill, 1978, p. 331]:

$$
\frac{\rho E c_{g_{2}}}{\omega}=\text { const }
$$

where $\rho$ is density, and $c_{g_{z}}=\partial \omega / \partial m$ is the vertical component of the group velocity. With (7) this can be rewritten in terms of the vertical velocity amplitude to describe the change in $A_{w}$ with height,

$$
A_{w}^{2}=A_{w_{0}}^{2} \frac{\rho_{0}}{\rho}\left[\frac{\left(N_{0}^{2} / \omega_{0}^{2}\right)-1}{\left(N^{2} / \omega^{2}\right)-1}\right]^{1 / 2} .
$$

The zero subscript refers to the lower boundary value. At some altitude this predicted amplitude will grow to the point where the wave would become unstable. Here the convective instability criterion is used to determine this wave breaking level, and the wave amplitude above that height is maintained at or below this instability limit. This is the traditional saturation theory, reviewed by Fritts [1984]. At the breaking level the total Eulerian parcel velocity exceeds the ground relative phase speed, and linear theory breaks down. Dewan and Good [1986] derive the instability criterion for the vertical velocity,

$$
A_{w}^{2} \leq \frac{\omega^{2}}{m^{2}}
$$

which after substitution of (11) to eliminate the vertical wavenumber can be written as

$$
A_{w}^{2} \leq \frac{\omega^{2}}{k^{2}\left[\left(N^{2} / \omega^{2}\right)-1\right]} .
$$

The breaking level of the wave is defined as that level where the amplitude equals or exceeds this saturation limit. Above that level, wave amplitudes will be modified as necessary by condition (14), and (13) is then taken to describe the linear growth in amplitude from one level (subscript zero) to the next. Waves that are reflected before reaching the breaking level are discarded and will have no net effect on the mean state. If saturation were to occur below the turning point, the wave would contribute to the mean state interaction only between the breaking level and the turning point, al- though this situation never occurs in the cases considered here.

The saturation condition (14) implies a certain shape of the power spectrum as a function of vertical wavenumber, $m$. VanZandt [1982] and many other authors since have described the universality of the shape of the corresponding horizontal velocity power spectrum $P_{u}(m)$ in observations, which follows a power law approximately proportional to $\mathrm{m}^{-3}$. For the vertical velocity the ana$\log$ to equation (2) for the vertical wavenumber power spectrum and (14) imply the saturated power spectral density,

$$
P_{w}(m)=\frac{\frac{1}{2} A_{w}^{2}}{(\Delta m)}=\frac{\omega^{2}}{2 m^{2} \Delta m}
$$

where $\Delta m$ is the spectral bandwidth. The linear dispersion relation (11) simplifies in the hydrostatic approximation $\left(m^{2}>>k^{2}\right)$ to

$$
\omega^{2}=\frac{N^{2} k^{2}}{m^{2}}
$$

which is a good approximation in the short vertical wavelength "tail" of the spectrum where saturation occurs. Substitution then gives

$$
P_{w}(m)=\frac{N^{2} k^{2}}{2 m^{4} \Delta m} .
$$

The analogous expression for the saturated horizontal velocity power spectrum was derived by Dewan and Good [1986] and, with the definition of the vertical velocity power spectrum (analogous to (2)), can be written as

$$
P_{u}(m)=\frac{\frac{1}{2} A_{u}^{2}}{(\Delta m)}=\frac{N^{2}}{2 m^{2} \Delta m} .
$$

Note, however, that unlike the Dewan and Good [1986] analysis, no separate assumption about the bandwidth is made here to determine the shape of the saturated spectrum as a function of vertical wavenumber, $m$. The bandwidth, $\Delta m$, in this case, is dictated by the resolution $(\Delta k, \Delta \omega)$ of the power spectrum (Figure 2) used to define a mode in this analysis. The hydrostatic dispersion relation implies

$$
\Delta m=\frac{\Delta k}{k} m \mp \frac{\Delta \omega}{N k} m^{2}
$$

with $\Delta k$ and $\Delta \omega$ fixed constants in this case. Therefore the Dewan and Good [1986] assumption, $\Delta m \propto m$, will only apply in those regions of the spectrum where the first term on the right-hand side of (17) dominates. More generally, the saturated spectra in these calculations will follow $P_{w}(m) \propto m^{-5}$ to $m^{-6}$ and $P_{u}(m) \propto$ $m^{-3}$ to $m^{-4}$. Numerous spectral observations of horizontal wind fluctuations show that a $P_{u}(m) \propto m^{-3}$ spectral shape is ubiquitous in the middle atmosphere [VanZandt, 1982; Smith et al., 1987]. This observed power law relationship and the associated amplitude limits have been attributed to the process of saturation. The spectra produced in this model are roughly consistent with observations but do not provide any new 
insights into the origin of the observed spectral features. An example of the saturated $P_{u}(m)$ spectrum produced in this model will be shown in section 5 .

\subsection{Energy, Momentum, and Wave Action Fluxes}

Wave amplitudes derived from equations (13) and (14) can be used to compute the fluxes of energy and momentum associated with the convectively generated waves. These relationships have been derived by Fritts and VanZandt [1993] and reproduced here in slightly different form, in terms of the information contained in the power spectrum (Figure 2). By using equations (7) and (11), energy flux per unit density can be written as

$$
F_{E}=c_{g_{s}} E= \pm \frac{\omega}{k}\left(\frac{N^{2}}{\omega^{2}}-1\right)^{1 / 2} A_{w}^{2}
$$

Momentum flux per unit density is computed as the $x$ and $t$-averaged product of horizontal and vertical velocity perturbations, $\overline{u w}$, and with ( 8 ) becomes

$$
F_{P}=\frac{\left(A_{u}^{2} A_{w}^{2}\right)^{1 / 2}}{2}= \pm \frac{1}{2}\left(\frac{N^{2}}{\omega^{2}}-1\right)^{1 / 2} A_{w}^{2} .
$$

These expression differ from those of Fritts and VanZandt in the neglect of rotation effects $(f=0)$ and in that no "anisotropy factor" appears here because (18) and (19) describe a single wave mode rather than a full wave spectrum. Also unlike the work of Fritts and VanZandt, no assumptions about the shape of the spectrum are made to integrate these expressions over all frequencies and wavenumbers. Instead the integration is performed by summing over the individual points in the spectrum. This method allows the spectrum from our peculiar storm source to be free to take any shape. The Fritts and VanZandt model is instead intended to capture the more general properties of middle atmosphere waves.

An expression for wave action flux in terms of the same variables can also be derived using equations (7) and (11),

$$
F_{\mathrm{A}}=\frac{E}{\omega} c_{g_{\mathbf{2}}}=\left(\frac{N^{2}}{\omega^{2}}-1\right)^{1 / 2} \frac{A_{w}^{2}}{k}
$$

Equations (18), (19), and (20) describe fluxes associated with a single wave mode defined by $(k, \omega)$. Before computing net effects of the full spectrum of waves, the amplitude adjustments previously applied in (3) for the purpose of predicting breaking levels and saturation effects must first be removed. This amounts to dividing the amplitudes by the correction factors $\left(N_{x} N_{t} / n_{x} n_{t}\right)$, or

$$
F_{P_{h \omega}} \rightarrow F_{P_{k \omega}}\left(\frac{n_{x} n_{t}}{N_{x} N_{t}}\right)_{k \omega}^{2} \text {. }
$$

The same factor must also be applied to the energy and wave action fluxes before any net effects can be computed.

\subsection{Wave Interactions With the Mean State}

The fluxes derived above can be used to infer the effects of wave dissipation on the mean state. A vertical gradient in the momentum flux (19) implies a drag force per unit mass on the mean flow $U$ :

$$
D_{x}=-\frac{1}{\bar{\rho}} \frac{\partial}{\partial z}\left(\bar{\rho} F_{P}\right)
$$

if the momentum flux is averaged over a suitably large horizonal domain. ( $\bar{\rho}$ is the basic state density profile.) Integration over the spectrum

$$
\sum_{k, \omega}\left(D_{x}\right)_{k \omega}
$$

gives the net zonal drag on the mean flow, averaged over a distance of $384 \mathrm{~km}$ and a time interval of 4 hours, corresponding to the averaging over the full $(x, t)$ domain of the original spectrum.

Durran [1995] underscores the difficulty in using (22) to describe the momentum budget in numerical models with a limited horizontal domain. In particular, contributions to the full nonlinear momentum budget due to differences in the upstream and downstream values of $\left(\rho u^{2}+p\right)$ are significant. The net mean flow acceleration associated with breaking mountain waves in his model is spread over very large distances away from the region containing the largest wave perturbations. The same difficulties arise in evaluating the momentum budget in the nonlinear storm model.

With this in mind, the mean flow effects derived from (22) in the linear analysis described here are further normalized to represent an average over the circumference of the earth at a latitude of $40^{\circ}$ and over 1 day of time. The result given in $\mathrm{m} \mathrm{s}^{-1}$ day $^{-1}$ represents the zonally averaged drag exerted on the mean flow, and averaged over a day's time, due to one storm active for a period of 4 hours. This normalization also allows more direct comparison of these results to global parameterizations of gravity wave drag.

Gravity wave dissipation also leads to transfer of energy to the mean flow. The energy dissipation rate can be derived from conservation of wave action flux. By defining the wave action per unit density $\mathbf{A}=E / \omega$ and the associated flux of $\mathbf{A}, F_{\mathbf{A}}=\mathbf{A} c_{g_{z}}$, then

$$
\frac{\partial}{\partial t}(\rho \mathbf{A})=\frac{\partial}{\partial z}\left(\rho F_{\mathbf{A}}\right)
$$

Note that both sides of (23) are zero in the absence of wave dissipation. Solving for the energy dissipation rate, $\epsilon$,

$$
\epsilon \equiv-\frac{\partial E}{\partial t}=\frac{-\omega}{\rho} \frac{\partial}{\partial z}\left(\rho F_{\mathrm{A}}\right)
$$

describes the rate of energy transfer from the waves to the mean flow along a ray and with equation $(20)$ can be computed readily from the linear analysis. Analogous to the net zonal drag calculation, contributions from individual modes are summed to compute the net energy 
dissipation rate and normalized as described above. So the results then given in $\mathrm{m}^{2} \mathrm{~s}^{-3}$ represent the zonally averaged rate of energy dissipation to the mean state, averaged over a day's time, due to waves produced by a single storm active over a 4-hour period.

\section{Comparison of Nonlinear and Linear Models}

As a test of the validity of the method and assumptions within the analysis described in sections 2 and 3 , comparisons between these linear wave propagation calculations and the full nonlinear model between 13and $32-\mathrm{km}$ altitude are presented in this section. Vertical velocity $w(x)$, momentum flux profiles, and the power spectrum versus vertical wavenumber $P_{w}(m)$ are each computed from the linear model for comparison to the full nonlinear results. Ray paths and breaking level predictions from the linear model are also examined for qualitative agreement with the nonlinear model results.

\subsection{Reconstruction of the Vertical Velocity $w(x)$}

By using only the power spectrum (Figure 2) and the results of the linear wave propagation analysis, realizations of the field $w(x)$ at selected heights can be reconstructed and compared to instantaneous distributions of $w(x)$ in the nonlinear model. These reconstructions represent properties averaged in time. The comparison provides a test of the derived wave packet widths in $x$, as well as the overall amplitudes implied by the wave packet width adjustments in $\boldsymbol{x}$.

The ray tracing analysis provides the location of each point in the spectrum $X\left(z, k_{i}, \omega_{j}\right)$ via equations $(9)$ and (12). Ray paths in a stratosphere with constant $16 \mathrm{~m}$ $\mathrm{s}^{-1}$ winds, like those in the nonlinear simulation, are straight lines and form a simple fan pattern emanating from the central source region. The fan pattern appearance is similar to the pattern observable in the nonlinear model, but the time dependence of the wave forcing, especially evident in the eastward propagating waves (see Figure 1b), is not captured in this analysis. The ray location at a specified altitude is taken as the central locus of a packet of wave energy with amplitude determined by wave action flux conservation/saturation (equations (13) and (14)). The energy in the packet is then distributed in $x$ within a half-cosine envelope with wavelength $\pi x_{W P}$. This envelope was illustrated in Figure 5 and has the same amplitude and total energy of the square wave packet with width $x_{W P}$. Each mode is then renormalized by multiplication of the temporal width factor given by (6), returning the power back to time-averaged values. The bias factor used to estimate the true power at the peaks in the spectrum $\left(N_{x} N_{t} / n_{x} n_{t}\right)$ is also removed before the contributions from each spectral mode are summed to produce profiles of $w(x)$ shown in Figure 7 for an altitude of 30 $\mathrm{km}$. Since the sum of the contributions from each mode may depend strongly on the phase of the wave within each wave packet, the phase is randomized before the
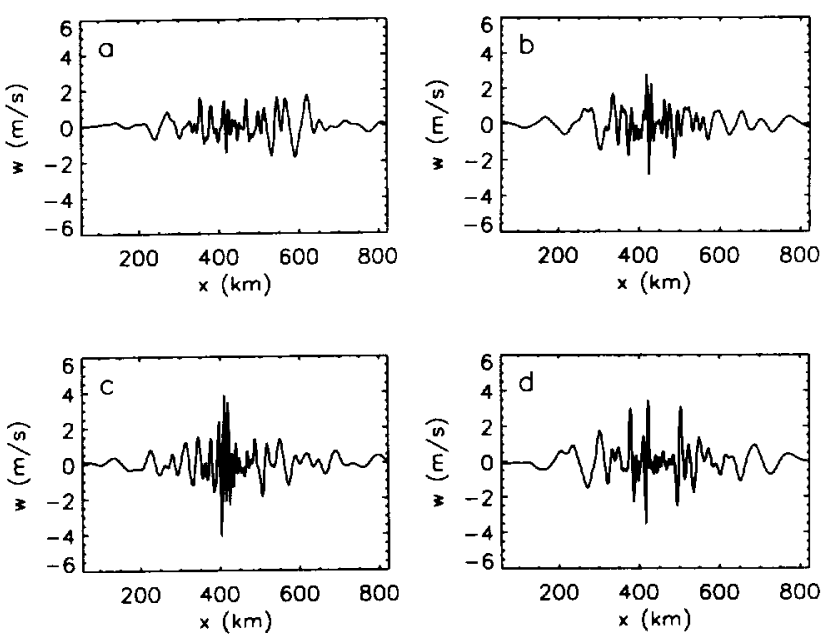

Figure 7. Reconstructions of the vertical velocity distribution $w(x)$ at $z=30 \mathrm{~km}$ from the spectral input and linear wave propagation analysis. (a)-(d) Different realizations of the field created by selecting different phase randomizations for each wave mode contributing to the total. The mean flow $U=0 \mathrm{~m} \mathrm{~s}^{-1}$ in these calculations so that results are shown in the storm-relative frame for comparison to the nonlinear model (Figure 8).

sum is computed. Four different realizations of the field $w(x)$ are presented in Figures $7 \mathrm{a}-7 \mathrm{~d}$, each computed with a different random number sequence to determine the phase randomization. Figures $8 \mathrm{a}-8 \mathrm{~d}$ show four realizations of the nonlinear model $w(x)$ at $30 \mathrm{~km}$. Figures 7 and 8 show similar amplitudes and similar variations as a function of $x$. The similarities suggest that the wave packet width in $x$ is approximately correct and that the computed ray paths are capturing the trajectories of the wave energy packets fairly well (despite the many simplifying assumptions like the point source approximation at $13 \mathrm{~km}$ and the time-averaging effect of using the power spectrum as input). Similar comparisons at $20 \mathrm{~km}$ (not shown) demonstrate that the growth of wave amplitude with height is being accurately simulated by the linear calculation; however, the distributions in $x$ appear less similar to the nonlinear model at this altitude much closer to the prescribed source (at $13 \mathrm{~km}$ ), presumably because of the inaccuracy of the point-source approximation.

\subsection{Momentum Flux Profiles}

Time- and $x$-averaged profiles of momentum flux can also be computed from the linear wave propagation analysis and compared to the nonlinear simulation. The nonlinear results are averaged over 4 hours and \pm 400 $\mathrm{km}$ from the central source region, with east and west halves of the domain averaged separately. The results are shown in Figure 9a. Momentum flux in the linear model can be calculated from equation (19), and contributions from each mode summed after removal of the wave packet and bias adjustments (21). Where ray paths extend beyond $\pm 400 \mathrm{~km}$ from the source, those waves do not contribute to the average for the purpose 

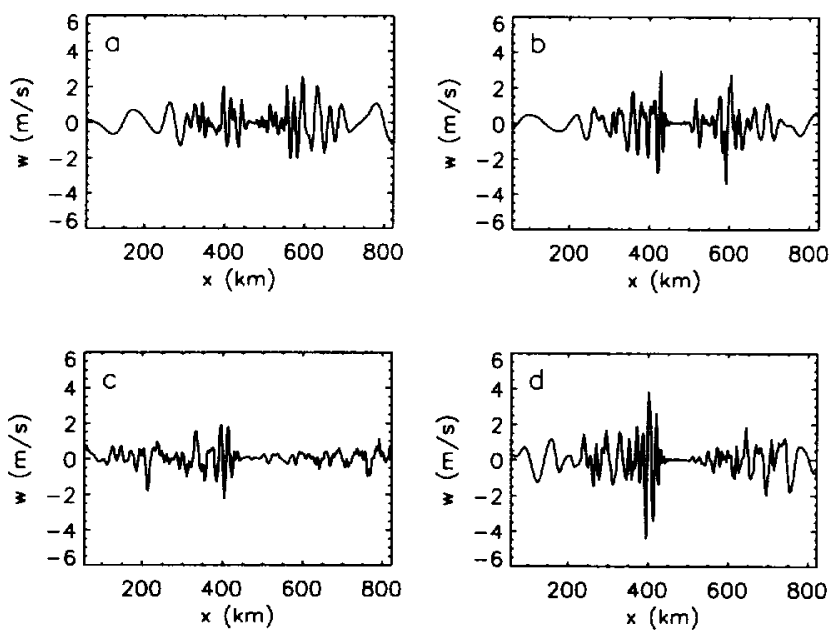

Figure 8. Vertical velocity distribution $w(x)$ at $z=30$ $\mathrm{km}$ from the nonlinear convection simulation. (a) $-(\mathrm{d})$ Four times $(3.03,3.53,6.03$, and 4.87 hours) selected randomly for comparison to the four reconstructions in Figure 7. The comparison shows very similar amplitudes, as well as similarities in the distribution of horizontal wavelength in $x$. This comparison provides a check on the computed ray paths and on the wave packet widths in the linear analysis.

of comparison to the nonlinear model. Westward and eastward propagating modes are averaged separately and overplotted in Figure 9a as heavy lines. The linear and nonlinear results are very similar at $z \geq 15$ $\mathrm{km}$, suggesting the momentum in the vertically propagating waves is well represented by the linear spectral model. At lower altitudes the nonlinear model shows an abrupt decrease in magnitude from the tropopause to $15 \mathrm{~km}$ in the westward average. This may be an indication of wave dissipation in the nonlinear model at these altitudes, where a thin layer of shear develops that is not considered in the linear comparison. The very low frequency waves not resolved in the spectrum may also be contributing to the domain-averaged momentum flux at these lower altitudes in the nonlinear model. Nonetheless, Figure 9a suggests the flux to the upper stratosphere and mesosphere is well characterized by the linear approximation.

A further test of the linear analysis is shown in Figure $9 \mathrm{~b}$. The thin lines again show momentum fluxes in a nonlinear simulation identical to that of $\mathrm{AHD}$, except for the inclusion of strong shear equal to $-2 \mathrm{~m} \mathrm{~s}^{-1} \mathrm{~km}^{-1}$ in the stratospheric winds above $13 \mathrm{~km}$. The stratospheric shear dramatically alters the vertically propagating waves but has no discernible effect on the development of the storm. The heavy lines in Figure 9b display the momentum flux profiles predicted from the original wave spectrum (Figure 2) and the linear wave propagation analysis through a background wind with shear identical to the nonlinear model. The linear and nonlinear momentum flux profiles are very similar in both magnitude and form. Differences are comparable to the no shear case in Figure 9a. Predicted ray paths also compare quite well to the nonlinear result (not shown). The comparison lends further credence to the linear wave treatment, even in this case with fairly strong background shear.

\subsection{Reconstruction of the Vertical Wavenumber Power Spectrum}

As a further test of the linear model assumptions the spectrum $P_{w}(m)$ can be computed from the spectrum in Figure 2, $P_{w}(k, \omega)$, with the help of the linear gravity wave dispersion relation (11). The result can be compared to the spectrum computed directly from the vertical velocity field in the nonlinear simulation without stratospheric shear. That spectrum, described by AHD is reproduced in Figure 10 and compared to the linear model reconstruction.

For the reconstruction the value of $m$ at each point in the $P(k, \omega)$ spectrum is computed from (11). The
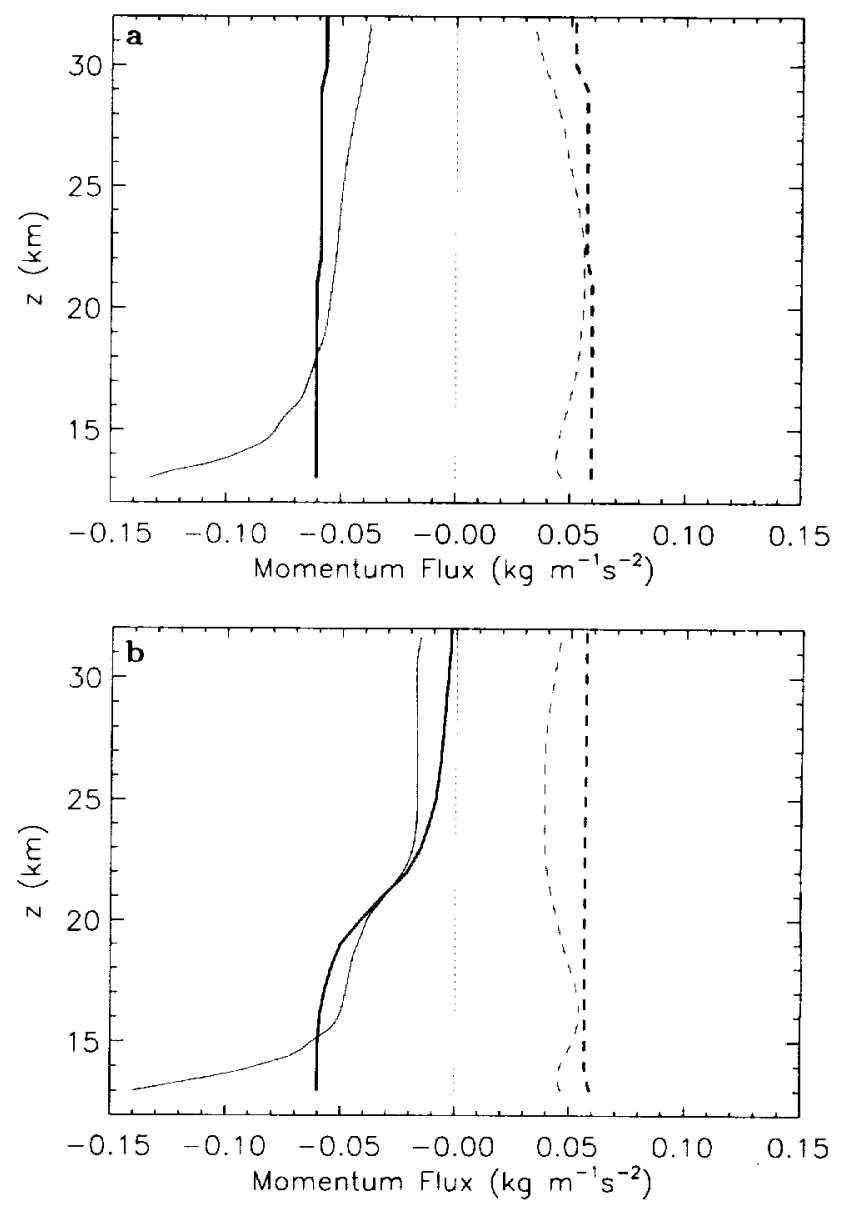

Figure 9. Profiles of momentum flux $\rho_{0} \overline{u w}$ averaged in $x$ and $t$. Thin lines show profiles averaged west (solid) and east (dashed) of storm center from the nonlinear convection simulation. Heavy lines show the same quantities reconstructed from the spectral input with the results of the linear ray tracing model. (a) Comparison of the AHD model and the linear analysis. (b) Comparison to a nonlinear simulation identical to AHD except with $-2 \mathrm{~m} \mathrm{~s}^{-1} \mathrm{~km}^{-1}$ shear in the stratosphere above 13 $\mathrm{km}$. The thin lines are derived from the same spectral input as in Figure 9a but are now affected by the strong background shear. 


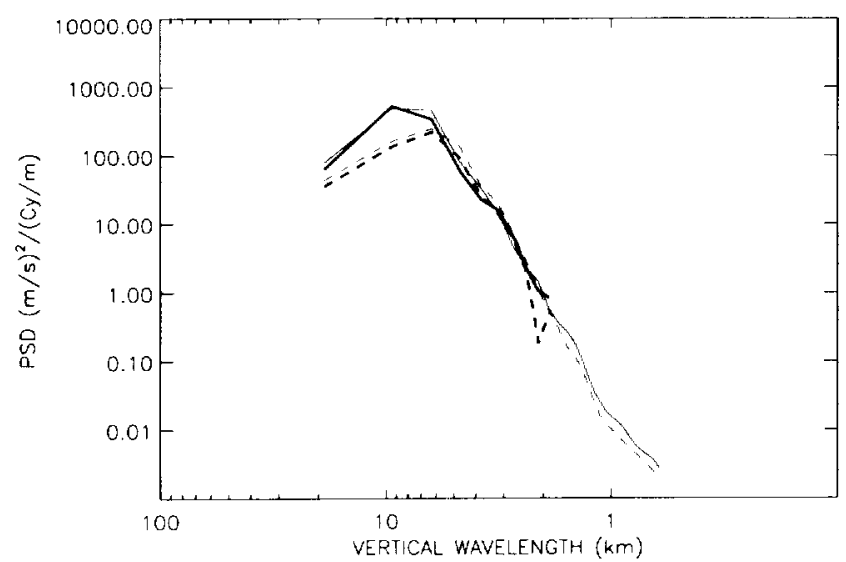

Figure 10. Power spectrum of the vertical velocity as a function of vertical wavenumber $P_{w}(m)$. Thin lines show spectra computed directly from the nonlinear convection simulation (AHD), while heavy lines show $\boldsymbol{P}_{\boldsymbol{w}}(m)$ reconstructed from the spectral input (Figure 2) by using linear gravity wave theory. This is the no-shear case. The solid and dashed lines represent the spectrum of waves west and east of storm center, respectively.

power at each point $P(k, \omega)$ is then binned in a vertical wavenumber array, identical to that in the original spectrum:

$$
m=\frac{n}{N_{z} \Delta z} \quad n=0,1, \ldots, \frac{N_{z}}{2}-1
$$

where $N_{z}=128$, and $\Delta z=148 \mathrm{~m}$ (AHD). Power falling into each bin is integrated over $k$ and $\omega$ :

$$
P(m) \Delta m=\sum_{k, \omega} P(k(m), \omega(m)) \Delta k \Delta \omega
$$

$P(m)$ is the resulting spectrum overplotted in Figure 10. The similarity of the result to the nonlinear model spectrum reflects the accuracy of applying linear gravity wave theory to these motions. It also suggests that the errors associated with truncating the power spectrum at $P_{\min }$ are small but evident as an absence of power in the low-energy tail of the reconstructed $P_{w}(m)$ spectrum.

\section{Wave Interactions With Realistic Background Wind Profiles}

With wave properties obtained from the nonlinear convection simulation, the linear ray tracing and wave action conservation model can predict the behavior of these vertically propagating waves through climatological background wind and buoyancy frequency profiles. The CIRA [Fleming et al., 1990] model at $40^{\circ} \mathrm{N}$ latitude provides background state profiles for this analysis. Profiles for April and June monthly averages are chosen to illustrate the nature of the wave-mean flow interactions.

\subsection{Breaking Levels and Critical Level Filtering} Associated With Variable Background Winds

The intrinsic frequency in the linear dispersion relation (11) will vary with height when there is shear in the background wind as $\omega=\omega_{0}-k U(z)$, so in the presence of shear, vertically propagating waves may find critical levels as described in section 3. This Doppler shifting also affects wave amplitude growth, breaking levels, and saturation of the waves via the frequency dependences in equations (13) and (14).

Figures $11 \mathrm{a}$ and $11 \mathrm{~b}$ illustrate some of these effects in the linear model. The solid lines represent climatological wind profiles for April and June. Symbols mark the ground-relative phase speeds of the waves in the spectrum along the abcissa. Dot symbols mark turning point altitudes where waves are reflected as the magnitude of their intrinsic frequency reaches the local buoy-
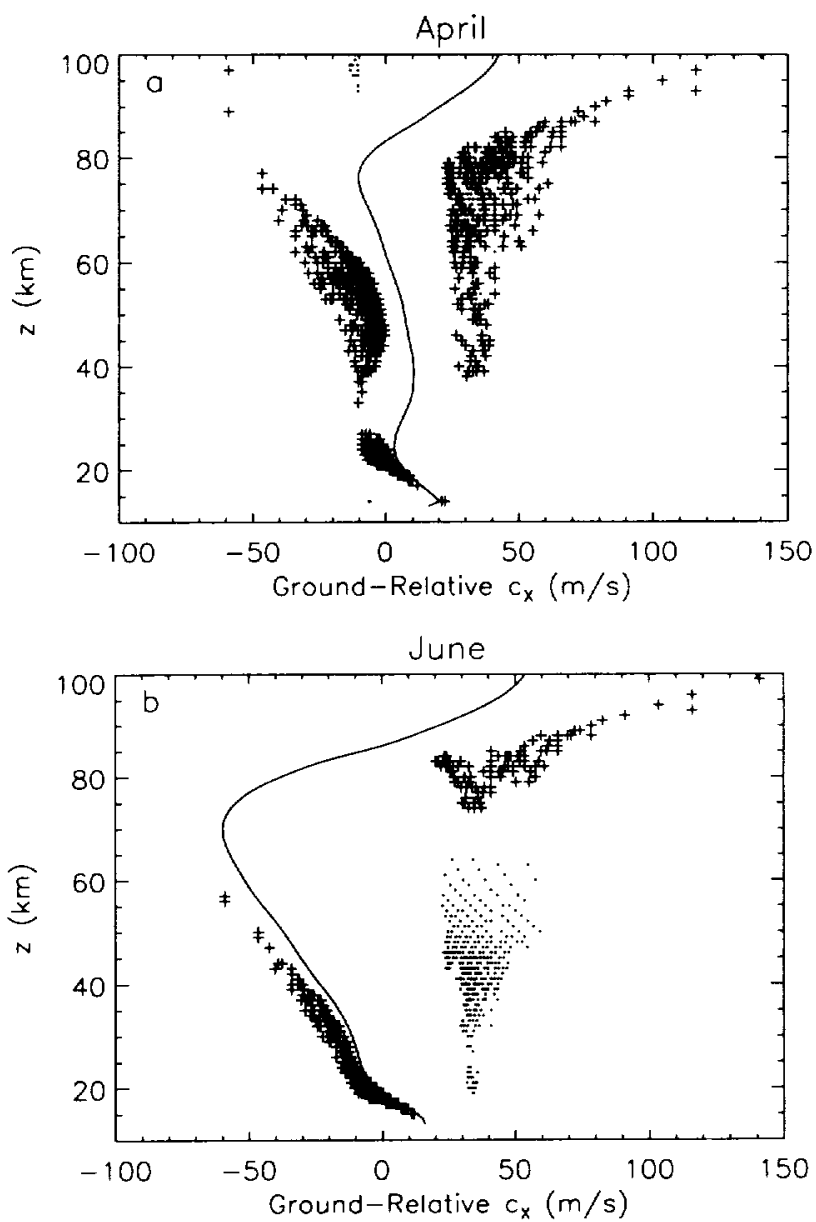

Figure 11. Zonal winds, breaking levels, and turning points for (a) April and (b) June, with background states defined by the CIRA model at $40^{\circ} \mathrm{N}$ latitude. Solid lines are zonal wind profiles $(U)$. Plus symbols mark breaking levels as a function of ground-relative phase speed $\left(c_{x}\right)$ for each wave mode. Dots denote turning points. Critical levels are not explicitly shown but occur at altitudes where $c_{x}=U$. Wave modes contribute to the mean flow forcing between their breaking and critical levels. 
ancy frequency. Wave reflection tends to occur in regions where the background shear causes growth in the intrinsic wave frequency. For the climatological winds considered here, none of these modes saturate below their reflection levels, so they are discarded from further analysis, having no net effect on the background state. Plus symbols mark breaking level altitudes as a function of phase speed. Wave breaking tends to occur where the background shear causes the intrinsic frequency to shrink in magnitude. If the shear is strong, as in the June case in the stratosphere, the breaking level tends to appear just below the critical level where $\omega \rightarrow 0$. This also tends to be true in the stratosphere where wave amplitudes predicted from wave action conservation (13) have not grown large enough to saturate (14) until their intrinsic frequencies become very small. In the much rarer atmosphere in the mesosphere, waves more often break well below any critical level and so contribute far more energy and momentum to the mean state forcing via the effects of saturation. Wave critical levels are not explicity shown but occur where the ground-relative phase speed matches the speed of the background wind.

\subsection{Saturation Effects on the Power Spectrum Versus Vertical Wavenumber}

The effects of saturation on the shape of the horizontal velocity perturbation power spectrum versus vertical wavenumber have been described by numerous authors [Dewan and Good, 1986; Smith et al., 1987; Fritts and VanZandt, 1993] and have been offered as an explanation for the ubiquitous observations of a power curve with a shape proportional to $\mathrm{m}^{-3}$ as described in section 3. Fritts and VanZandt [1993] describe this saturated spectrum as

$$
P_{u}(m)=\frac{(p+1)}{20} \frac{N^{2}}{m^{3}}
$$

with $p=\frac{5}{3}$. This theory also explains the nearly constant amplitude of the spectrum with height despite the large changes in density from the troposphere to the thermosphere. In regions where waves are saturating, the linear wave propagation model developed here displays characteristics similar to the theory and to spectral observations.

The region between $\sim 20$ and $30 \mathrm{~km}$ in the June case (Figure 11b) provides an example. At these altitudes, waves with phase speeds between $\sim-20$ and $0 \mathrm{~m} \mathrm{~s}^{-1}$ break, and their intrinsic frequencies shrink rapidly toward zero as the waves approach their critical levels. Alternatively, waves with phase speeds larger than 16 $\mathrm{m} \mathrm{s}^{-1}$ are Doppler shifted to higher frequency. The effect of the shear on the vertical wavenumber spectrum is shown in Figure 12. The thin line shows the original spectrum, $P_{u}(m)$, at $13-\mathrm{km}$ altitude. Waves with phase speeds of $<16 \mathrm{~m} \mathrm{~s}^{-1}$ are Doppler shifted to higher vertical wavenumbers and saturate. The eastward propagating waves with phase speeds of $>16 \mathrm{~m} \mathrm{~s}^{-1}$ are Doppler shifted to lower $m$ and grow in amplitude

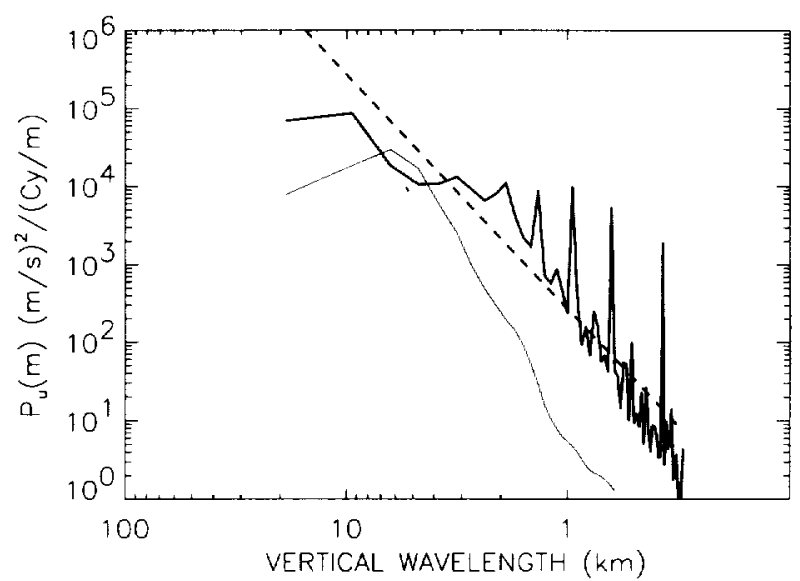

Figure 12. Saturation effects on the horizontal velocity power spectrum $P_{u}(m)$. The thin line shows the input wave spectrum at $13 \mathrm{~km}$ including both eastward and westward propagating waves. The heavy solid line shows the "saturated spectrum" between 20 and $30 \mathrm{~km}$ in the June case. Eastward propagating waves are Doppler shifted to long $\lambda_{z}$ and grown in amplitude, while westward waves get shifted to short $\lambda_{z}$ and saturate. The dashed line is the theoretical curve, equation (25).

as they propagate vertically. The saturated spectrum averaged between 20 and $30 \mathrm{~km}$ is shown as the thick solid line in Figure 12, and the theoretical saturation limit (25) is plotted as a dashed line. The spectrum is noisy, since it is computed as a sum of the discrete set of modes, but approximately follows an $\mathrm{m}^{-3}$ to $\mathrm{m}^{-4}$ power law, as expected from equations (16) and (17), and the amplitude is similar to that given by the theory (25). (Note that variations in wave packet travel times associated with variations in group velocity are neglected in these calculations.) It should be emphasized that the fit of the $P_{u}(m)$ spectrum in this model to the saturation theory (25) is a direct result of the spectral treatment (17) coupled to the imposed saturation condition (16). This result provides no new insight into the physical mechanisms responsible for the observed $\mathrm{m}^{-3}$ power law. It has been suggested that wave-wave interactions in fact produce the observed spectral shape [Weinstock, 1990; Hines, 1991], and these have been neglected in the present model. Figure 12 shows, however, that the linear spectral model with saturation described here is at least not inconsistent with the observations.

\subsection{Zonal Drag and Dissipation Rates}

Wave drag and dissipation rates for the April and June cases are shown in Figures 13 and 14 . The rates plotted represent the contribution to the mean flow acceleration or wave energy dissipation rate resulting from the single storm of 4-hour duration averaged over the latitude circle at $40^{\circ} \mathrm{N}$ and over a day's time. Also shown for reference are the parameterization results from Fritts and $L u$ [1993] plotted at one-tenth magnitude as dashed lines. In both figures, lower panels display stratospheric altitudes and upper panels show mesospheric altitudes. 

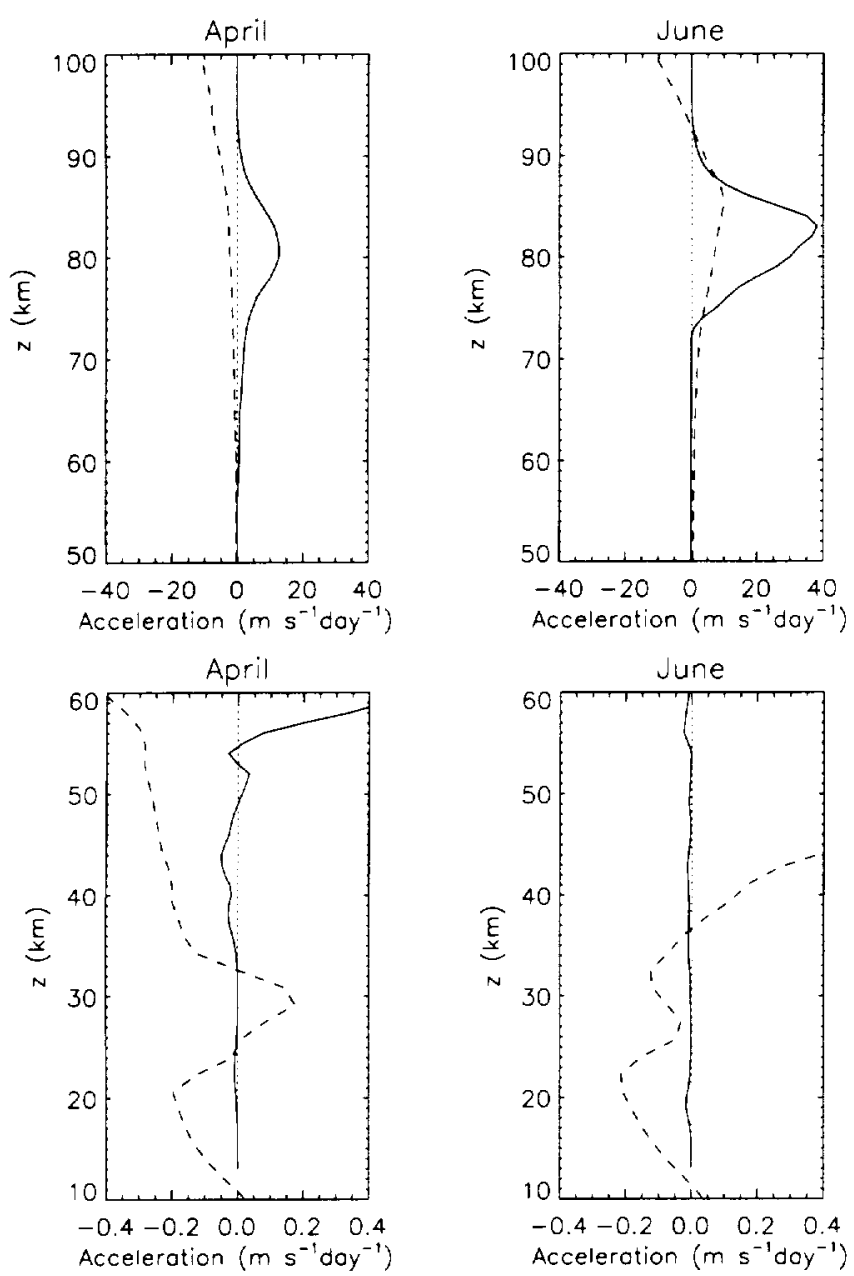

Figure 13. Zonal mean drag force per unit mass on the zonal wind from the convectively generated wave spectrum (solid lines). The dashed lines show the Fritts and $L u$ [1993] parameterization at one tenth the magnitude for comparison. Upper panels show mesospheric altitudes, while lower panels show the stratospheric results at finer scale.

The storm forcing is not very similar in form to the parameterization. These high-frequency waves, forced in the upper troposphere, have only weak effects in the lower stratosphere. In the more rarified upper stratosphere and mesosphere their effects can be substantial. The storm-generated wave effects dwindle in the lower thermosphere: Most of the original spectrum has been reflected or absorbed at critical levels below these altitudes.

In the June case, stratospheric shear in the mean winds eliminates much of the spectrum carrying negative momentum flux (waves with $c_{x}<16 \mathrm{~m} \mathrm{~s}^{-1}$ ). These waves are absorbed at critical levels in the stratosphere (Figure 11b). The critical level absorption, however, contributes little to the mean flow forcing (Figures 13 and 14), because as the intrinsic frequency approaches zero at the critical level, so the wave energy also goes to zero, conserving wave action flux. Wave saturation due to the density effect has a far greater effect on the mean state. In the mesosphere, in this case, waves carry- ing eastward momentum flux $\left(c_{x}>16 \mathrm{~m} \mathrm{~s}^{-1}\right)$ saturate well below their critical levels and deposit substantial momentum and energy at these levels. The Fritts and Lu parameterization predicts a peak drag of $\sim 100 \mathrm{~m}$ $\mathrm{s}^{-1}$ day $^{-1}$ at $85 \mathrm{~km}$ in general agreement with Holton's [1982] original summer soltice estimate. The storm drag peaks at nearly $40 \mathrm{~m} \mathrm{~s}^{-1}$ day $^{-1}$ and so is a substantial fraction of the zonal mean in the upper mesosphere. In April the shear is weaker, and the storm-generated waves create drag of opposite sign as that of Fritts and $\mathrm{Lu}$ in the mesosphere. Referring to Figure 11a, the waves that are breaking above $60 \mathrm{~km}$ have eastward phase speeds, so they produce an eastward drag force. The parameterized drag, on the other hand, has some tendency to oppose the mean wind regardless of such phase speed preferences.

The largest uncertainty in these results lies in the determination of wave packet widths that influence where the waves break. To evaluate this uncertainty, wave packet widths defined by the $95 \%$ confidence limits on the straight line fits in Figure 6 are alternately substituted for equations (5) and (6), and the wave/mean
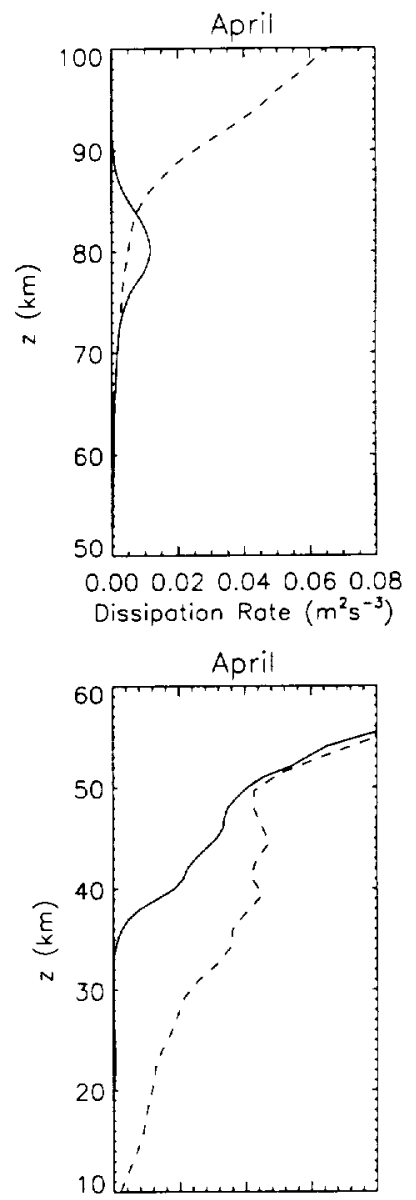

0.000 .050 .100 .150 .20 Dissipotion Rate $\left(10^{-3} \mathrm{~m}^{2} \mathrm{~s}^{-3}\right)$
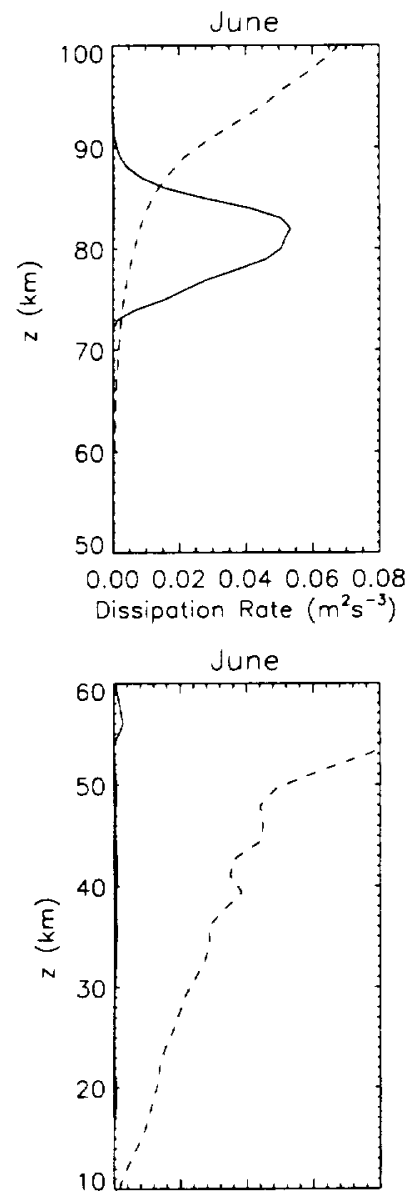

$0.00 \quad 0.05 \quad 0.10 \quad 0.15 \quad 0.20$ Dissipation Rote $\left(10^{-3} \mathrm{~m}^{2} \mathrm{~s}^{-3}\right)$
Figure 14. Wave energy dissipation rates from the convectively generated wave spectrum (solid lines) are compared to one tenth the magnitude of the Fritts and $L u$ [1993] parameterization (dashed lines). 

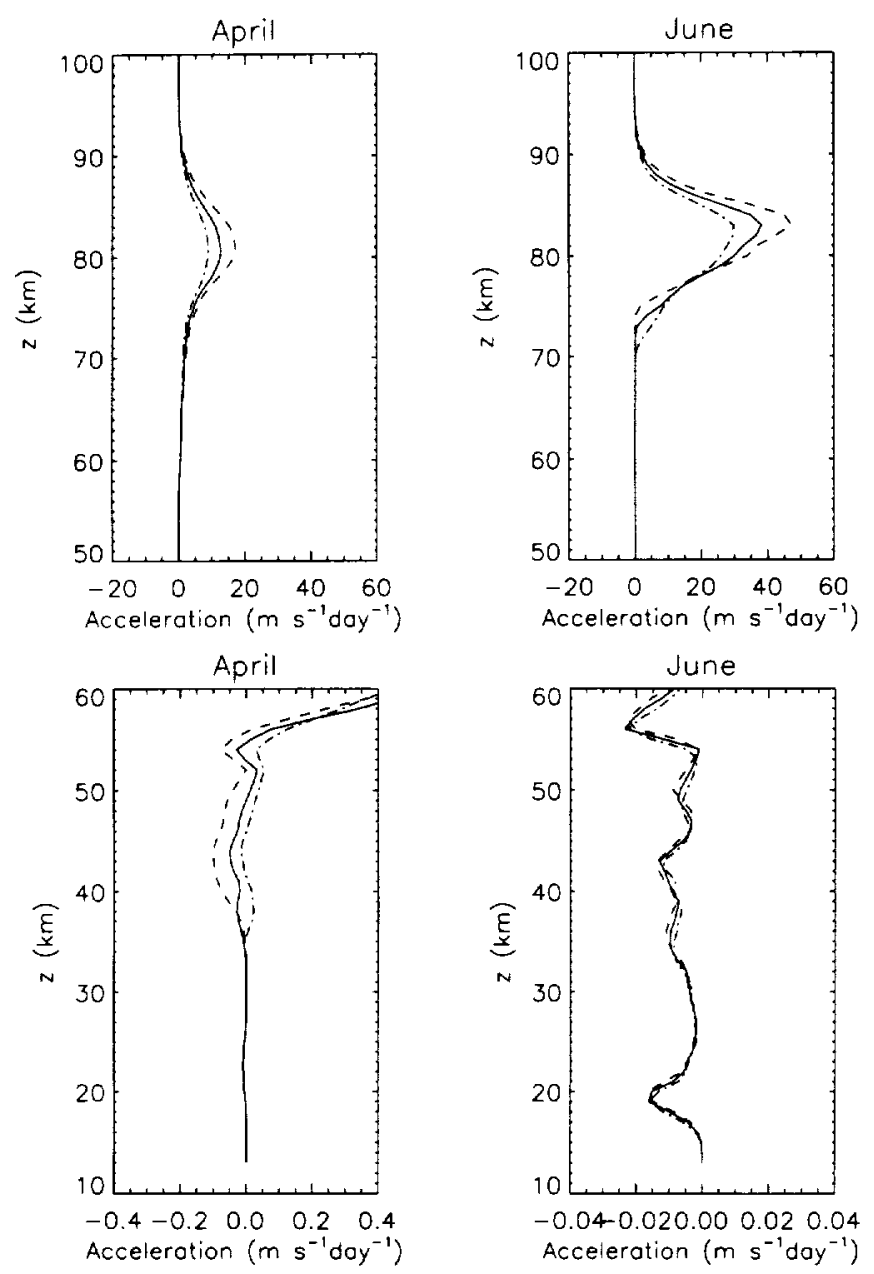
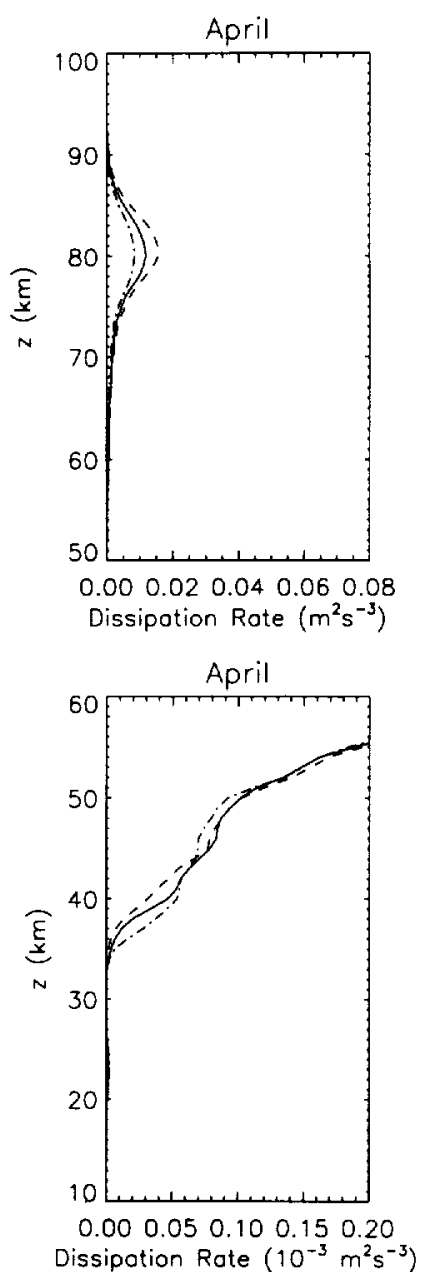
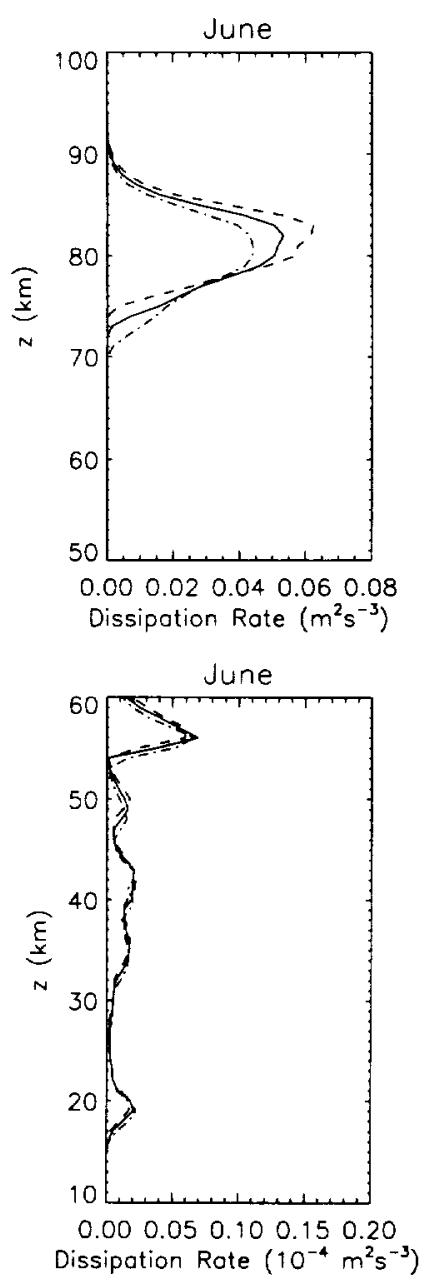

Figure 15. The effect of uncertainty in the wave energy packet widths on the mean flow acceleration. Solid lines are the same as those in Figure 14. Dashed lines show the effect of packet widths defined by the upper limit fits shown in Figure 6. Dot-dashed lines show effects of the lower limits.

interaction is recomputed. The results are shown in Figures 15 and 16. Note that these changes do not result in a simple scaling of the profiles in Figures 13 and 14. Instead the forcing is redistributed in $z$. The changes affect the breaking levels of the waves but not the net energy and momentum they carry at the lower boundary, which are fixed by the integrated power in the input spectrum. The changes can be significant at some altitudes but do not affect the overall conclusions. Still this uncertainty underscores the need for caution in the quantiative use of the forcing profiles derived in this analysis.

\subsection{Mesopause Perturbations}

Figure 17 shows estimates of temperature perturbations at the mesopause constructed in a manner analogous to the vertical velocities in Figure 7 . Temperature perturbations are derived from the wind perturbations via the polarization relations [Gossard and Hooke, 1975] and the ideal gas equation of state. Here, $x=0$

Figure 16. The effect of uncertainty in the wave energy packet widths on the energy dissipation rate profile. Solid lines are the same as those in Figure 15. Dashed lines show the effect of packet widths defined by the upper limit fits shown in Figure 6. Dot-dashed lines show effects of the lower limits.
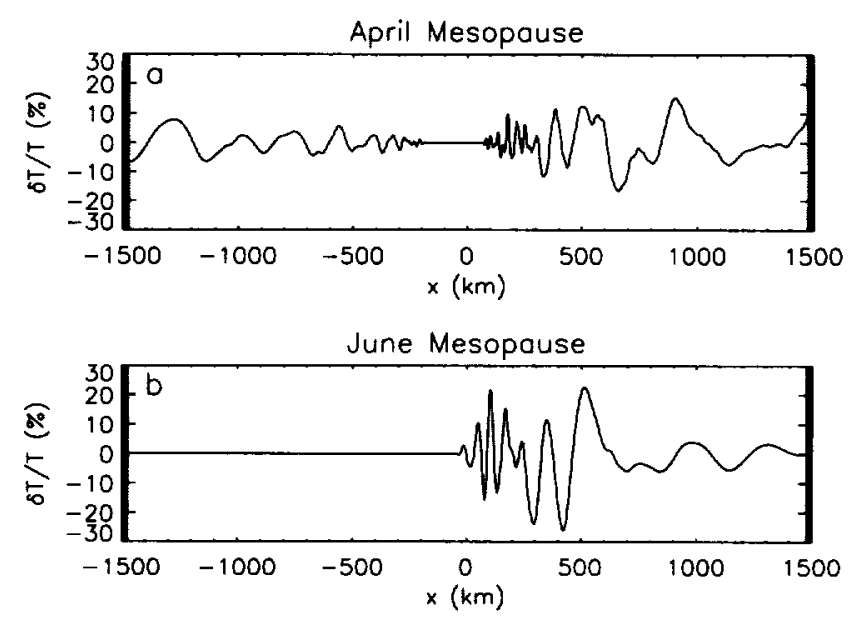

Figure 17. Mesopause temperature perturbation reconstructions, $\delta T(x)$, shown as percent of the mesopause temperature for the (a) April and (b) June cases. 
represents the location of the wave source specified at $13 \mathrm{~km}$. Filtering of the westward propagating waves by wind shear is particularly evident in the June case, where there is an absence of wave perturbations west of the storm source. Amplitudes to the east of the source are limited by saturation in this region, where positive shear in the zonal wind is present for both the April and June cases. Peak perturbations are approximately $10-$ $15 \%$ in the April case, and $20-30 \%$ for June. Vertical wavelengths at the mesopause for these waves cluster in the 5- to $20-\mathrm{km}$ range for April, 10 - to $30-\mathrm{km}$ for June. These are large perturbations, and they would be readily visible in observations of the $\mathrm{OH}$ airglow like those reported by Swenson and Mende [1994] and Taylor et al. [1995]. A pattern of increasing horizontal wavelength with increasing distance from the source region is a common feature of these calculations and is consistent with the aircraft-based observations reported by Swenson and Mende [1994] in the vicinity of a Pacific storm system. Radiative damping, not included in this analysis, might reduce the amplitude of the perturbations derived here. Rough calculations using the scale dependent damping rates of Fels [1984] suggest that amplitudes in the layer between 55 and $85 \mathrm{~km}$ would be reduced by only $1 \%$ or less over most of the spectrum, but a few of the modes may be significantly damped $(\sim 10 \%)$.

\section{Summary and Conclusions}

This paper describes a technique for evaluating the effects of simulated convectively generated gravity waves on the middle atmosphere. The method employs linear gravity wave theory in a ray tracing calculation from the tropopause to the lower thermosphere. Conservation of wave action flux predicts wave amplitude growth with height along the rays, and a saturation condition derived from the convective instability criterion is employed to predict breaking levels and limits to the amplitude growth.

In the work of AHD an analysis of the gravity wave spectrum observed above the deep convection in their squall line simulation established that some of the defining characteristics of the spectrum result directly from the wave-forcing mechanisms active in the model and that some of these characteristics have also been seen in ground-based observations of motions above deep convective storms. The spectrum of simulated waves above the storm AHD describe provides the lower boundary input to the ray tracing model. Input wave amplitudes as a function of frequency and horizontal wavenumber are determined by combining the results of Fourier and wavelet spectral analysis techniques. The Fourier analysis provides a high-resolution two-dimensional spectrum of wave amplitudes as a function of frequency and wavenumber, but these amplitudes are averaged over the entire spatial/temporal interval of the analysis. Since the wave modes observed in the convection simulation are not uniformly present in space and time, an estimate of the distribution of wave energy is pro- vided by the spatial and temporal information retained in an orthogonal wavelet analysis, although at greatly reduced spectral resolution. This information on the distribution in $(x, t)$ of the spectral energy $E(k, \omega)$ is cast into an estimate of the wave energy packet widths in space and time and then used to adjust the amplitudes in the power spectrum derived from Fourier analysis to represent local, rather than averaged, values. The adjusted amplitudes allow realistic estimates of wave breaking levels in the ray tracing calculation; however, the wave packet adjustments are removed before evaluation of any net wave/mean state interactions to properly conserve energy. The model is tested for consistency with the full nonlinear simulation below 32 $\mathrm{km}$, where they overlap.

This ray tracing model is subsequently used to examine the effects of realistic mean wind profiles on the convectively generated gravity wave spectrum. The effects of variable buoyancy frequency are also included. Critical level absorption, wave reflection, and the effects of Doppler shifting and saturation on the power spectrum versus vertical wavenumber are all treated in fairly realistic manner and produce results consistent with observations and theory.

The results are cast in terms of the drag force per unit mass on the mean flow and wave energy dissipation rate and compared to the Fritts and $L u$ [1993] parameterization of gravity wave effects. The comparison suggests that single large storm systems could contribute significant fractions of estimated zonal mean wave forcing at some altitudes, particularly in the mesosphere. Further theoretical work on gravity waves generated by deep convection will establish the generality of these conclusions.

This model may also be useful in assessing relationships between storm generated waves and observations of gravity wave induced brightness variations in the high altitude airglow such as those obtained during the recent ALOHA 93 campaign and reported by Swenson and Mende [1994]. Predicted temperature fluctuations at mesopause altitudes derived from this theoretical analysis would be easily detectable in such $\mathrm{OH}$ airglow observations, and some of the characteristics of waves associated with the Pacific storm in the observations are qualitatively matched in these theoretical results.

The model developed here also provides a means of comparing other convectively generated gravity wave simulations and may allow the assessment of the relationship between general characteristics of convectively generated waves and their global effects on the middle atmosphere. It can also be used to test simpler parameterizations of convectively generated waves against calculations with more detailed spectral information such as the example in this work.

Acknowledgments. The author would like to thank Jim Holton for his insightful comments on the manuscript and for donating his numerical modeling results that went in to preparing Figure 9b. Dave Fritts provided lots of guidance in developing a working version of the Fritts-VanZandtLu gravity wave parameterization. Norman McFarlane and 
Julio Bacmeister also contributed thought-provoking comments and suggestions that improved the manuscript considerably. This work was supported by grants from the National Science Foundation, ATM-8813971, and NASA, NAGW-662.

\section{References}

Alexander, M. J., J. R. Holton, and D. R. Durran, The gravity wave response above deep convection in a squall line simulation, J. Atmos. Sci., 52, 2212-2226, 1995.

Bacmeister, J. T., Mountain-wave drag in the stratosphere and mesosphere inferred from observed winds and a simple mountain-wave parameterization scheme, J. Atmos. Sci., 50, 377-399, 1993.

Dewan, E. M., and R. E. Good, Saturation and the "universal" spectrum for vertical profiles of horizontal scalar winds in the atmosphere, J. Geophys. Res., 91, 2742-2748, 1986.

Dunkerton, T. J., Theory of the mesopause semiannual oscillation, J. Atmos. Sci., 99, 2681-2690, 1982.

Durran, D. R., Do breaking mountain waves decelerate the local mean flow?, J. Atmos. Sci., in press, 1995.

Eckermann, S. D., Ray-tracing simulation of the global propagation of inertia gravity waves through the zonally averaged middle atmosphere, J. Geophys. Res., 97, 15,849$15,866,1992$.

Fels, S. B., The radiative damping of short vertical scale waves in the mesosphere, $J$. Atmos. Sci., 41, 1755-1764, 1984.

Fleming, E.L., S. Chandra, J.J. Barnett, and M. Corney, Zonal mean temperature, pressure, zonal wind and geopotential height as functions of latitude, Adv. Space Res., 10(12), 11-59, 1990.

Fovell, R., D. Durran, and J. R. Holton, Numerical simulations of convectively generated stratospheric gravity waves, J. Atmos. Sci., 49, 1427-1442, 1992.

Fritts, D. C., Gravity wave saturation in the middle atmosphere: A review of theory and observations, Rev. Geophys., 28, 275-308, 1984.

Fritts, D. C., and T. E. VanZandt, Spectral estimates of gravity wave energy and momentum fluxes. Part I: Energy dissipation, acceleration, and constraints, J. Atmos. Sci., 50, 3865-3694, 1993.

Fritts, D. C., and W. Lu, Spectral estimates of gravity wave energy and momentum fluxes. Part II: Parameterization of wave forcing and variability, J. Atmos. Sci., 50, 3695$3713,1993$.

Garcia, R. R., and S. Solomon, The effect of breaking gravity waves on the dynamics and chemical composition of the mesosphere and lower thermosphere, J. Geophys. Res., 90, 3850-3860, 1985.

Gossard, E.E., and W.H. Hooke, Waves in the Atmosphere, 456 pp., Elsevier, New York, 1975.

Hines, C. O., The saturation of gravity wres in the middle atmosphere. Part II: Development of Doppler-spread theory, J. Atmos. Sci., 48, 1360-1379, 1991.

Holton, J. R., The role of gravity wave induced drag and diffusion in the momentum budget of the mesosphere, J. Atmos. Sci., 39, 791-799, 1982.

Holton, J. R., The influence of gravity wave breaking on the general circulation of the middle atmosphere, J. Atmos. Sci., $40,2497-2507,1983$.

Holton, J. R., and D. Durran, Convectively generated stratospheric gravity waves: The role of mean wind shear, in
Coupling Processes in the Lower and Middle Atmosphere, edited by E. V. Thrane et al., pp. 175-189, Kluwer Acad., Norwell, Mass., 1993.

Houze, R. A., Jr., Cloud Dynamics, pp. 348-394, Academic Press, San Diego, Calif., 1993.

Larsen, M. F., W. E. Swartz, and R. F. Woodman, Gravity-wave generation by thunderstorms observed with a vertically-pointing $430 \mathrm{MHz}$ radar, Geophys. Res. Lett., 9, 571-574, 1982.

Lighthill, J., Waves in Fluids, 504 pp., Cambridge Univ. Press, New York, 1978.

Lindzen, R. S., Turbulence and stress owing to gravity wave and tidal breakdown, J. Geophys. Res., 86, 9707-9714, 1981.

Marks, C. J., and S. D. Eckermann, A three-dimensional nonhydrostatic ray-tracing model for gravity waves: Formulation and preliminary results for the middle atmosphere, J. Atmos. Sci., 52, 1959-1984, 1995.

Matsuno, T., A quasi one-dimensional model of the middle atmosphere circulation interacting with internal gravity waves, J. Meteorol. Soc. Jpn., 60, 215-226, 1982.

McFarlane, N. A., The effect of orographically excited gravity wave drag on the general circulation of the lower stratosphere and troposphere, J. Atmos. Sci., 44, 1775$1800,1987$.

Palmer, T. N., G. J. Shutts and R. Swinbank, Alleviation of a systematic westerly bias in general circulation and numerical weather prediction models through an orographic gravity wave drag parameterization, $Q$. J. R. Meteorol. Soc., 112, 1001-1039, 1986.

Percival, D. B., and A. T. Walden, Spectral Analysis for Physical Applications: Multitaper and Conventional Univariate Techniques, 583 pp., Cambridge Univ. Press, New York, 1993.

Press, W. H., B. P. Flannery, S. A. Teukolsky, W. T. Vetterling, Numerical Recipes: The Art of Scientific Computing, 2nd ed., 702 pp., Cambridge Univ. Press, New York, 1993.

Sato, K., Small-scale wind disturbances observed by the MU radar during the passage of typhoon Kelly, J. Atmos. Sci., 50, 518-537, 1993.

Smith, S. A., D. C. Fritts, and T. E. VanZandt, Evidence for a saturated spectrum of atmospheric gravity waves, J. Atmos. Sci., 44, 1404-1410, 1987.

Swenson, G., and S. Mende, Atmospheric airglow imaging with CCDs, Proc. SPIE Int. Soc. Opt. Eng., 2266-15, 1994.

Taylor, M. J., V. Taylor, and R. Edwards, An investigation of thunderstorms as a source of short period mesospheric gravity waves, in The Upper Mesosphere and Lower Thermosphere: A Review of Experiment and Theory, Geophys. Monog. Ser., 87, edited by R. M. Johnson and T. L. Killeen, pp. 177-184, AGU, Washington, D.C., 1995.

VanZandt, T. E., A universal spectrum of buoyancy waves in the atmosphere, Geophys. Res. Lett., 9, 575-578, 1982.

Weinstock, J., Saturated and unsaturated spectra of gravity waves and scale-dependent diffusion, J. Atmos. Sci., 47 , 2211-2225, 1990.

M. J. Alexander, University of Washington, Department of Atmospheric Sciences, Box 351640, Seattle, WA 981951640 .

(Received December 7, 1994; revised May 25, 1995; accepted May 25, 1995.) 